\title{
Cellular Senescence and Senescence-Associated Secretory Phenotype Drive Multipotent Cardiac Stem/Progenitor Cell Dysfunction in Human Diabetic Cardiomyopathy Independently of Age
}

\section{Fabiola Marino}

Magna Graecia University of Catanzaro: Universita degli Studi Magna Graecia di Catanzaro

Mariangela Scalise

Magna Graecia University of Catanzaro: Universita degli Studi Magna Graecia di Catanzaro

Donato Cappetta

University of Campania Luigi Vanvitelli: Universita degli Studi della Campania Luigi Vanvitelli

Luca Salerno

Magna Graecia University of Catanzaro: Universita degli Studi Magna Graecia di Catanzaro

Claudia Molinaro

Magna Graecia University of Catanzaro: Universita degli Studi Magna Graecia di Catanzaro

\section{Nadia Salerno}

Magna Graecia University of Catanzaro: Universita degli Studi Magna Graecia di Catanzaro

\section{Michele Torella}

University of Campania Luigi Vanvitelli: Universita degli Studi della Campania Luigi Vanvitelli

\section{Marta Greco}

Magna Graecia University of Catanzaro: Universita degli Studi Magna Graecia di Catanzaro

\section{Daniela Foti}

Magna Graecia University of Catanzaro: Universita degli Studi Magna Graecia di Catanzaro

\section{Ferdinando C. Sasso}

University of Campania Luigi Vanvitelli: Universita degli Studi della Campania Luigi Vanvitelli

\section{Pasquale Mastroroberto}

Magna Graecia University of Catanzaro: Universita degli Studi Magna Graecia di Catanzaro

Antonella De Angelis

University of Campania Luigi Vanvitelli: Universita degli Studi della Campania Luigi Vanvitelli

Georgina M. Ellison-Hughes

King's College London

\section{Marcello Rota}

New York Medical College

\section{Konrad Urbanek}

Magna Graecia University of Catanzaro: Universita degli Studi Magna Graecia di Catanzaro 


\section{Daniele Torella ( $\boldsymbol{\sim}$ dtorella@unicz.it)}

Magna Graecia University of Catanzaro: Universita degli Studi Magna Graecia di Catanzaro https://orcid.org/0000-0002-4915-5084

\section{Eleonora Cianflone}

Magna Graecia University of Catanzaro: Universita degli Studi Magna Graecia di Catanzaro

\section{Research Article}

Keywords: Senescence, Senescence-Associated Secretory Phenotype, Diabetes, Aging, Cardiac Stem/Progenitor Cells

Posted Date: August 4th, 2021

DOl: https://doi.org/10.21203/rs.3.rs-607324/v1

License: (c) (i) This work is licensed under a Creative Commons Attribution 4.0 International License. Read Full License 


\section{Abstract}

Background: Aging and Diabetes Mellitus (DM) independently and additively increase cardiovascular risk and a pathophysiological basis for this epidemiological link is currently the subject of intense investigations. Both aging and DM affect the biology and regenerative potential of tissue specific cardiac adult stem/progenitor cells (CSCs). In aged subjects over half of the CSCs are senescent with a senescence-associated secretory phenotype (SASP) that renders otherwise healthy CSCs to senescence, impairing their proliferative and differentiation potential. Although a link to organismal aging is clear, cells can undergo senescence, regardless of age. Yet, it is unclear whether the SASP is induced by Diabetes per se in CSCs and whether targeting senescent cells within the diabetic CSC compartment rescues their regenerative biology.

Methods: In order to investigate the effects of DM on CSC senescence and to try separate those of aging from Diabetes on senescence events, we obtained peri-infarct/border zone biopsies from non-aged patients (50-64 years old) with DM type 2 (T2DM) and non-diabetic (NDM) patients with post-infarct cardiomyopathy undergoing surgical coronary revascularization.

Results: Ischemic injury in DM is associated with a higher ROS production, as revealed by the exacerbated expression of 8-OH-deoxyguanosine, nitrotyrosine, and 4-hydroxinonenal targeting both cardiomyocytes as well as CSCs. The latter associated with an increased number of senescent and dysfunctional T2DM-hCSCs (isolated from atrial samples of T2DM and NDM patients with ischemic cardiomyopathy) identified by increased $\mathrm{p} 16^{\text {INK4a }}$ positive cells, reduced telomerase activity and telomere length, reduced proliferation, clonogenesis/spherogenesis and myogenic differentiation when compared to NDM-hCSCs in vitro. Importantly, T2DM-hCSCs show a defined SASP, as demonstrated by the increased secretion of MMP-3, PAI1, IL-6, IL-8, IL-1 $\beta$ and GM-CSF. A combination of two senolytics, Dasatinib and Quercetin, clears senescent T2DM-hCSCs in vitro restoring expansion and myogenic differentiation capacities of the diabetic hCSC pool.

Conclusions: DM hampers human CSC biology, inducing a variety of hallmarks of senescence in nonaged subjects that contribute to the deficit of their regenerative potential. Clearance of senescent cells by senolytics abrogates the SASP and restores a fully proliferative- and differentiation- competent hCSC pool in T2DM.

\section{Background}

Aging is a major risk factor for the occurrence of several diseases, including stroke, myocardial infarction, heart failure, neurodegenerative disease, and several cancers. Therefore, the global rise

in life expectancy will lead to a dramatic increase in age related diseases in the coming decades. In this scenario, cardiovascular diseases including atherosclerosis and heart failure (HF) increase exponentially with age, whereby HF is considered an epidemic because of a growing and ageing population. Type 2 diabetes mellitus (T2DM) is closely bound to aging and is a powerful independent risk factor for 
cardiovascular diseases such as atherosclerosis and heart failure [1]. This epidemiological connection between aging, T2DM, and cardiovascular diseases postulates that there might be a pathophysiological link.

Aging has been associated with systemic inflammation and oxidative stress [2], which can be both a cause as well as a consequence of Diabetes [3]. Cellular senescence can be defined as a permanent arrest of cellular growth and is a key feature of aging $[4,5]$. Cell senescence is also a cause and a consequence of Diabetes and plays an important role in its cardiovascular complications. Although senescent cells are classically reported as cell that irreversibly cease proliferation, they have the capacity to produce and secrete soluble factors that can influence neighboring cells and tissues [6, 7]. This feature of senescent cells to secrete these soluble factors has taken the name of senescence-associated secretory phenotype (SASP) [8]. Since chronic inflammation is an important pathophysiological factor of both aging and diabetes, the SASP has been pointed as the pathophysiological link between aging and diabetes in cardiovascular diseases [9].

Tissue-specific adult stem cell senescence has emerged as an attractive theory for the decline in mammalian tissue and organ function during aging [10]. The mammalian heart, including the human, harbors a tissue specific cardiac stem/progenitor cell (CSC) compartment [11-15] that undergoes senescence with age, which dictates a progressive and permanent dysfunction of more than half of these endogenous cells by 75 years of age $[5,10,16,17]$. The senescent CSCs exhibit a SASP that can negatively impact surrounding cells, causing otherwise healthy and cycling-competent CSCs to lose proliferative capacity and switch to a senescent phenotype. Nevertheless, even at the oldest age, it is still possible to retrieve a healthy, cycling-competent CSC fraction with an increased regenerative and reparative capacity $[5,10,16]$. Accordingly, experimental selective ablation of senescent CSCs either genetically or by a combination of senolytic drugs, fosters the expansion and functional regenerative recovery of the healthy aged CSCs $[5,10,18]$.

Several reports have shown that Diabetes impairs the in vitro proliferative and differentiation potential of adult CSCs, further worsening their senescence phenotype even when compared with CSCs from nondiabetic ischemic patients [19-21]. Changes in chromatin conformation underlie the impaired proliferation, differentiation, and senescent behavior of diabetic CSC [22]. Yet, it is unclear whether the SASP is induced by Diabetes per se in CSCs and whether targeting senescent cells with the diabetic CSC compartment rescues CSC proliferation and differentiation defect in T2DM. On this premise here we show that the myocardium of T2DM patients, in a very narrow age window of 55-64 years, undergoing cardiac revascularization for ischemic heart disease, is characterized by an increased oxidative stress that affects the CSC compartment with an increased senescent phenotype when compared to myocardium and CSCs of age and sex matched NON-Diabetic Mellitus (NDM) patients. Importantly, Diabetic-Senescent CSCs have a SASP and senolityc treatment abrogate the senescent diabetic DiabeticSenescent CSCs uncaging healthy CSCs to proliferate and differentiate normally.

\section{Methods}




\section{Patients Cohort and Samples}

Human myocardial samples were obtained from Type 2 diabetes mellitus (T2DM) and NON-Diabetic Mellitus (NDM) patients with post-infarct cardiomyopathy which undergoing surgical coronary revascularization. The samples were collected only when removal of tissue was required for surgical reasons. Collection of human tissues samples was approved by the local ethics committee at the University of Campania "L. Vanvitelli" of Naples. Before cardiac surgery, written informed consent was obtained from every patient. All patient data were kept anonymous. We included 10 T2DM and 6 NDM patients from which peri-infarct/border zone biopsies were obtained (Table 1). Freshly excised samples were formalin fixed for immunohistochemistry analysis as described below. Furthermore, additional 6 T2DM and 6 NDM patients with similar characteristics were included (Table 1), from which atrial samples were obtained and processed for cell harvesting as below described.

\section{Human CSCs Isolation}

Human samples obtained from T2DM and NDM patients were stored in saline on ice until ready to process $(\sim 1 \mathrm{hr})$. All steps were performed at $4^{\circ} \mathrm{C}$ unless stated otherwise. Briefly, cardiac tissue was minced then digested with collagenase II $(0.3 \mathrm{mg} / \mathrm{ml}$; Worthington Laboratories) in Dulbecco's Modified Eagle's Medium (DMEM; Sigma-Aldrich) at $37^{\circ} \mathrm{C}$ in a series of sequential digestions for 3 minutes each. Enzymatically released cells were filtered through a $40 \mu \mathrm{m}$ cell strainer (Becton Dickinson, BD) to eliminate the cardiomyocyte population and collected in enzyme quenching media (DMEM + 10\% FBS). The isolated cardiac cells were collected by centrifugation at $400 \mathrm{~g}$ for $10 \mathrm{~min}$, resuspended in incubation media (PBS, $0.5 \%$ BSA, 2 mM EDTA). For the isolation of $c-k t^{\text {pos }} C D 45^{\text {neg }} C D 31^{\text {neg }} \mathrm{CSCs}$ the MACS technology was used (Miltenyi Biotec). First, cardiomyocyte-depleted cardiac cells were negatively sorted for $\mathrm{CD} 45^{\text {pos }}$ and $C D 31^{\text {pos }}$ cells by immunolabelling with anti-human CD45 and CD31 magnetic immunobeads (Miltenyi). The obtained CD $45^{\text {neg }} C D 31^{\text {neg }}$ population was then enriched for $\mathrm{c}$-kit ${ }^{\text {pos }}$ cardiac cells through incubation with anti-human CD117 immunobeads (Miltenyi) (1:10) and then positively sorted using MACS according to the manufacturer's instructions.

\section{Cell Culture}

Freshly isolated human c-kit ${ }^{\text {pos }} \mathrm{CD} 45^{\text {neg }} \mathrm{CD} 31^{\text {neg }} \mathrm{CSC}$ s were plated in CELL-Start (Life Technologies) coated dishes in complete CSC growth medium consisting of a 1:1 ratio of DMEM-F12-Ham's Gibco, Life Technologies) medium and Neurobasal medium (Gibco, Life Technologies), implemented with Embryonic stem-cell FBS (10\%, Gibco, Life Technologies), insulin-transferrin-selenium (1\%, Life Technologies), epidermal growth factor $(20 \mathrm{ng} / \mathrm{ml}$, Peprotech), basal fibroblast growth factor (10 ng/ml, Peprotech), human leukemia inhibitory factor (10 ng/ml, Miltenyi), $37 \mathrm{mg}$ of I-glutamine, B27 supplement ( $2 \%$, Life Technologies), N2 supplement (1\%, Life Technologies), penicillin-streptomycin (1\%, Life Technologies), Fungizone $(0.1 \%$, Life Technologies) and gentamicin ( $0.1 \%$, Life Technologies). Cells were maintained in a humidified hypoxic incubator at $37^{\circ} \mathrm{C}, 5 \% \mathrm{CO}_{2}, 2 \% \mathrm{O}_{2}$. Media were replenished every 48 hours and cells were passaged at a 1:4 ratio. 


\section{Histology and Immunohistochemistry}

Tissue specimens were fixed and embedded in paraffin for histochemical and immunohistochemical analysis. Human tissues were cut in sections of $5 \mu \mathrm{m}$. After dewaxing in xylene and rehydration in graded concentration of ethanol, when appropriate, Antigen Retrieval was performed by incubating section in 10 $\mathrm{mM}$ sodium citrate buffer $(\mathrm{pH} 6.0)$ at $98^{\circ} \mathrm{C}$ for $30 \mathrm{~min}$. Non-specific antibody binding was blocked by incubation with $10 \%$ normal donkey serum (Jackson ImmunoResearch) for 30 minutes at room temperature. Sections were stained for $1 \mathrm{~h}$ at $37^{\circ} \mathrm{C}$ or overnight at $4^{\circ} \mathrm{C}$ and the following primary antibodies were used: c-kit (1:100 dilution, DAKO), c-Tnl (1:200 dilution, Abcam), p16 ${ }^{\text {INK4A }}$ (1:100 dilution, Santa Cruz Biotechnology), 8-OH-dG (1:100 dilution, Origene) and 3-NT (1:400 dilution, Merck Millipore). After washing in phosphate-buffered saline (PBS), sections were incubated with respective secondary antibodies (Jackson ImmunoResearch). Nuclear counterstains were performed by DAPI (4',6-diamidino-2phenylindole, Sigma) and sections were examined by confocal microscopy (LEICA TCS SP8). The number of positive cells was expressed as a percent fraction of the total cells number per $\mathrm{mm}^{2}$.

For immunohistochemical analysis of oxidative stress on tissue sections, after dewaxing, human sections were blocked with Dual Endogenous Enzyme Block for $10 \mathrm{~min}$ at room temperature. Then, the sections were stained with antibodies against 8-OH-dG (1:100 dilution, Origene), 3-NT (1:400 dilution, Merck Millipore) and 4-HNE antibody (1:50 dilution, Abcam) for $1 \mathrm{~h}$ at $37^{\circ} \mathrm{C}$. Positive reactions were visualized using a Labelled Polymer-HRP complex and 3,3'-diaminobenzidine tetrahydrochloride (DAB) chromogen using the EnVision+ Dual Link System-HRP (DAKO). Sections were then counterstained with hematoxylin, permanently mounted, and then examined with light microscopy (LEICA, DMI3000B). The morphometric analysis of immunohistochemistry was conducted using Image $\mathrm{J}$.

\section{FACS analysis}

Cell analysis was performed on FACSCanto II (BD) with FlowJo software (TREE STAR) to identify the percentage of cardiac small cells expressing different cell-surface markers of interest at passage 2 (P2). A panel of different markers was used for immunophenotypic characterization of human ckit $^{\text {pos }}$ CD $45^{\text {neg }}$ CD $31^{\text {neg }}$ CSCs obtained from T2DM and NDM patients. Specific antibodies used are listed in Table 2. Appropriate labelled isotype controls were used to define the specific gates.

\section{Proliferation, clonogenicity, cardiosphere formation and cardiomyocyte differentiation assays in vitro}

CSC proliferation was evaluated through BrdU incorporation and growth curve assay, at the indicated time points, on human CSCs. To assess the proliferative activity of freshly isolated c$\mathrm{kit}^{\text {pos }}{ }^{\mathrm{CD}} 45^{\text {neg }} \mathrm{CD} 31^{\text {neg }}$ T2DM-hCSCs and NDM-hCSCs, BrdU $10 \mu \mathrm{M}$ was administered in vitro. CSCs were plated at density of $1 \times 10^{3}$ in 24-well CELL-Start-coated dishes and then serum starved in $0 \%$ serum base medium. After $48 \mathrm{hrs}$, starvation medium was replaced by CSC growth medium and BrdU was added to the medium every $6 \mathrm{hrs}$. The cells were fixed at 24 hours and BrdU incorporation was assessed using the 
BrdU detection system kit (Roche) according to the manufacturer's instructions. Nuclei were counterstained with DAPI (Sigma-Aldrich). Cells were evaluated using a fluorescent microscope (LEICA, DMI3000B). Accordingly, the number of BrdU ${ }^{\text {pos }}$ cells was expressed as a percent fraction of the total cell nuclei.

Growth curve assay was archived by plating $5 \times 10^{3}$ cells in 24-well CELL-Start-coated dishes in CSC growth medium and then serum starved in $0 \%$ serum base medium. After $48 \mathrm{hrs}$, starvation medium was replaced by CSC growth medium and cells were then trypsinized and counted using trypan blue, 1:1 ratio, at the indicated time points.

Single cell cloning was employed through depositing of half c-kit ${ }^{\text {pos }} C D 45^{\text {neg }} C D 31^{\text {neg }} T 2 D M-h C S C s$ and NDM-hCSCs into 96-well CELL-Start-coated Terasaki plates by serial dilution. Individual ckit $^{\text {pos }}$ CD $45^{\text {neg }} C D 31^{\text {neg }}$ T2DM-hCSCs and NDM-hCSCs were grown in CSC growth medium for 1-3 weeks when clones were identified and expanded. The clonogenicity of the c-kit ${ }^{\text {pos }} C D 45^{\text {neg }} C D 31^{\text {neg }} T 2 D M-$ hCSCs and NDM-hCSCs was determined by counting the number of wells in each 96-well plate containing clones and expressed as a percentage. A total of 10 plates were analyzed.

For cardiosphere generation, $1 \times 10^{5} \mathrm{c}-\mathrm{kit}^{\text {pos }} \mathrm{CD} 45^{\text {neg }} \mathrm{CD} 31^{\text {neg }} \mathrm{T} 2 \mathrm{DM}$-hCSCs and NDM-hCSCs were placed in bacteriological dishes with CSC growth medium. Cardiospheres were counted per plate at 14 days and the number expressed as a percentage relative to the number of plated CSCs.

For specific myogenic differentiation, c-kit ${ }^{\text {pos }} C D 45^{\text {neg }} C D 31^{\text {neg }}$ T2DM-hCSCs and NDM-hCSCs-derivedcardiospheres were switched to StemPro®-34 SFM differentiation medium (a serum-free medium conditioned with StemPro®-Nutrient Supplement, Gibco, Life Technologies), Glutamine (2mM) and penicillin-streptomycin (1\%, Life Technologies). For cardiomyocyte differentiation BMP4 (10ng/ml,

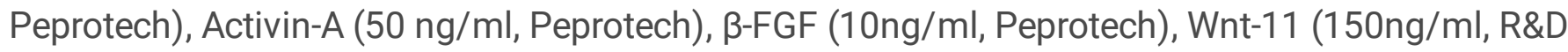
System) and Wnt-5a (150ng/ml, R\&D System) were added to base differentiation medium. Then differentiating cardiospheres were pelleted and transferred to laminin coated dishes $(1 \mu \mathrm{g} / \mathrm{ml})$ and Dkk-1 (150ng/ml, R\&D System) was added to base differentiation medium until day 14 . Cell differentiation was evaluated at 14 days.

\section{Immunocytochemistry}

A volume of $200 \mu \mathrm{l}$ of a $0.15 \times 10^{6}$ cells $/ \mathrm{ml}$ suspension of c-kit ${ }^{\text {pos }} \mathrm{CD} 45^{\text {neg }} \mathrm{CD} 31^{\text {neg }} \mathrm{CSC}$ s obtained from T2DM and NDM patients were directly loaded in cytofunnel and spin down at $800 \mathrm{rpm}$ for 3 min onto poly-lysine-coated slides using a Shandon Cytospin 4 Cytocentrifuge (Thermo Fisher Scientific). Slides were immediately fixed using PFA 4\% (Sigma-Aldrich). After fixation, cells were allowed to air dry before proceeding with immunostaining. Slides were washed with PBS, and incubated in 0.1\% Triton X-100:PBS at room temperature for 10 minutes. After washing with $0.1 \%$ tween:PBS and 30 minutes in $10 \%$ donkey serum, cells were incubated overnight at $4^{\circ} \mathrm{C}$ with primary antibodies against p16 ${ }^{\text {INK4a }}$ (1:100 dilution, Santa Cruz Biotechnology), $\mathrm{Y}-\mathrm{H} 2 \mathrm{AX}$ (1:400 dilution, Cell Signaling) in 0.1\% Tween:PBS. Slides were then 
washed and incubated with corresponding secondary antibodies (Jackson Immunoresearch) for 1 hour at $37^{\circ} \mathrm{C}$. After washing, the nuclear DNA of the cells was counterstained with DAPI (Sigma-Aldrich) at $1 \mu \mathrm{g} / \mathrm{ml}$ and mounted using Vectashield mounting media (Vector labs). Imagines were acquired using a confocal microscope (LEICA TCS SP8). The number of $\mathrm{p} 16^{\text {INK4a }}$ positive and $\mathrm{y}-\mathrm{H} 2 \mathrm{AX}$ positive cells was expressed as a percent fraction of the total CSC nuclei. Cell apoptosis were detected using the Terminal deoxynucleotidyl Transferase (TdT) assay (TUNEL assay, Merck Millipore) according to the manufacturer's instructions. Briefly, to perform TUNEL staining, cells were fixed with paraformaldehyde $2 \%$ in PBS ( $\mathrm{pH} 7.4$ ) and permeabilized with $0.1 \%$ Triton $\mathrm{X}-100$ in $0.1 \%$ sodium citrate. Then cells were incubated with TUNEL reaction mixture (containing Terminal deoxynucleotidyl transferase enzyme + nucleotide mixture in reaction buffer) for 1 hour at $37^{\circ} \mathrm{C}$ in a humidified atmosphere in the dark. After washing, the nuclear DNA of the cells was counterstained with DAPI (Sigma-Aldrich) and the slides mounted using mounting media (Vector labs). Imagines were acquired using a confocal microscope (LEICA TCS SP8). The number of apoptotic cells was expressed as percentage of the total nuclei.

SA- $\beta$-gal staining was performed using a SA- $\beta$-gal staining kit (Cell Signaling Technology) according to the manufacturer's instructions and protocols previously reported [23]. In brief, c-kit ${ }^{\text {pos }} C D 45^{\text {neg }} C D 31^{\text {neg }}$ T2DM-hCSCs and NDM-hCSCs were grown in culture and then fixed with 1X Fixative Solution for 10-15 min at room temperature. Following fixation, cells were washed with PBS before being incubated in $\beta$ Galactosidase Staining Solution ( $\mathrm{pH} \mathrm{6.0)}$ at $37^{\circ} \mathrm{C}$ in a dry incubator overnight. The enzymatic reaction was stopped by washing slides with ice-cold PBS and SA- $\beta$-gal staining was fixed with ice-cold methanol for 30 s before mounting/visualising. Senescent CSCs were identified as blue-stained cells using light microscopy. A minimum of 10 images were taken at $x 10$ magnification from random fields and the percentage of $S A-\beta$-gal cells were expressed as percentage of total nuclei.

To perform cTnl staining, human c-kit ${ }^{\text {pos }} \mathrm{CD} 45^{\text {neg }} \mathrm{CD} 31^{\text {neg }} \mathrm{CSC}$-derived cardiospheres were fixed using PFA 4\% (Sigma-Aldrich) after 14 days in differentiation media. After fixation, cells were incubated with cTnl antibody $\left(1: 200\right.$, Abcam) for 2 hour at $37^{\circ} \mathrm{C}$ in a humidified atmosphere. The corresponding secondary antibody (Jackson Immunoresearch) was added for 1 hour at $37^{\circ} \mathrm{C}$ in the dark. The nuclear DNA of the cells was counterstained with DAPI (Sigma-Aldrich) and the slides mounted using mounting media (Vector labs). Imagines were acquired using a confocal microscope (LEICA TCS SP8).

\section{Telomere length}

Genomic DNA of human c-kit ${ }^{\text {pos }} C D 45^{\text {neg }} C D 31^{\text {neg }}$ CSCs obtained from myocardial samples of T2DM and NDM patients was extracted using Quick-DNA Microprep Kit (Zymo Research). Telomere length was analyzed by using the Absolute Human Telomere Length Quantification qPCR Assay Kit (ScienCell Research Laboratories). Genomic DNA (10 ng) was amplified with the FastStart DNA Green Master (Roche Life Science) using a CFX384-Real-Time PCR System (Biorad) and data analysis was conducted according to manufacturer's instruction. For each genomic DNA samples, two different reaction were performed using two primer set: a Telomere primer set to recognize and amplify the telomere sequences and a single copy reference (SCR) primer set to normalize the data that recognizes and amplifies a 100 
bp-long region on human chromosome 17 , accordingly, a known telomere length as reference to calculate the telomere length of target samples. All reactions were run in triplicate. The average telomere length was calculated by following the manufacturer's instructions.

\section{Telomerase Activity Quantification}

Human c-kit ${ }^{\text {pos }} C D 45^{\text {neg }} C D 31^{\text {neg }} C S C s$ obtained from myocardial samples of T2DM and NDM patients were processed according to the manufacturer's protocol using the Telomerase Activity Quantification qPCR Assay Kit (ScienCell Research Laboratories) [24]. Briefly, cell pellets were thawed in lysis reagent enables to release telomerases in the native state. Cell lysate samples were incubated with telomerase reaction buffer at $37^{\circ} \mathrm{C}$ for 3 hours. qRT-PCR was conducted with FastStart DNA Green Master (Roche Life Science) using a CFX384-Real-Time PCR System (Biorad) and data analysis was performed according to manufacturer's instruction. The telomere primers set recognizes and amplifies newly synthesized telomere sequences in the assay. All reactions were run in triplicate. Telomerase activity quantification was calculated by following the manufacturer's instructions.

\section{RNA extraction and RT-PCR analysis}

RNA was extracted from human c-kit ${ }^{\text {pos }} C D 45^{\text {neg }} C D 31^{\text {neg }} C S C s$ obtained from myocardial samples of T2DM and NDM patients, using TRIzol ${ }^{\circledR}$ RNA Isolation Reagents (Ambion) and quantitated using a Nanodrop 2000 Spectrophotometer (Thermo Fisher scientific). Reverse transcription was performed with 0.5-1 $\mu \mathrm{g}$ of RNA using HighCapacity cDNA Kit (Applied Biosystem). Quantitative RT-PCR was performed using TaqMan Primer/Probe sets (see Table 3) using StepOne Plus real Time PCR System (Applied Biosystems). The following genes were tested: GATA-4, NKX2.5, MEF2C TNNT2, ACTC1, MYH6, MYH7, MCP1, IGFBP5, MMP3, IL-6, CCL11, IL-8, GM-CSF and PAI1. Data were processed by the $\triangle$ Ct method using StepOne Software v2.3 and mRNA was normalized to the housekeeping gene, GAPDH. All reactions were carried out in triplicate.

\section{Western Blot Analysis}

Immunoblots were carried out using protein lysates obtained from c-kit ${ }^{\text {pos }} C D 45^{\text {neg }} C D 31^{\text {neg }}$ T2DM-hCSCs and NDM-hCSCs. Aliquot equivalent of $\sim 40$ to $70 \mu \mathrm{g}$ of proteins were separated on gradient (6-15\%) SDS-polyacrylamide gels. After electrophoresis, proteins were transferred onto nitrocellulose filters, blocked with either $5 \%$ dry milk and incubated with Ab against p16 ${ }^{\text {INK4A }}(1: 1000$, Santa Cruz Biotechnology) and GAPDH (1:1000, Santa Cruz Biotechnology). Proteins were detected by chemiluminescence using horseradish peroxidase-conjugated $2 \mathrm{Abs}$ and placing the nitrocellulose filters on a photographic film. The acquisition was archived using Medical X-ray processor 2000 (CARESTREAM). Densitometry was obtained using ImageJ software. Immunoblots were performed in biological triplicates.

\section{Cytokine \& growth factors assay}


Cytokine and growth factor levels [Interleukin-1a (IL-1a), Interleukin-1 $\beta$ (IL-1ß), Interleukin-2 (IL-2), Interleukin-4 (IL-4), Interleukin-6 (IL-6), Interleukin-8 (IL-8), Interleukin-10 (IL-10), Interferon Y (IFNY), tumor necrosis factor alpha (TNFa), Monocyte Chemoattractant Protein-1(MCP-1), Vascular Endothelial Growth Factor (VEGF), Epidermal growth factor (EGF)] were simultaneously evaluated through the "Cytokine \& Growth Factors Array (CTK)" kit (Randox Labs) by using Evidence Investigator biochip analyzer (Randox Labs) [25], a chemiluminescent immunoassay in which antibodies bonded on the biochip surface allow the immobilization of a specific analyte, subsequently bonded with a secondary antibody labelled with horseradish peroxidase (HRP), to trigger a luminol-based chemiluminescent signal emission. The light signal generated from each of the test regions is then detected using imaging technology and quantified with a stored calibration curve.

\section{Senolytic drug treatment, viability in vitro}

c-kit ${ }^{\text {pos }} C D 45^{\text {neg }} C D 31^{\text {neg }} T 2 D M-h C S C S$ were plated in 24-well CELL-Start-coated plates at $40 \%$ confluence and left for $72 \mathrm{~h}$. Then, the growing medium was replaced with new fresh medium in the absence or presence of a combination of two senolytics drug, Dasatinib (D, LC Laboratories) and Quercetin ( $Q$, Sigma-Aldrich), respectively at doses $0.25 \mu \mathrm{M}$ and $10 \mu \mathrm{M}$ added for 6 hours. Then, the conditioned medium was replaced with complete fresh medium. After $48 \mathrm{~h}$ the treatment $\mathrm{D}+\mathrm{Q}$ was repeated as above.

For myogenic differentiation, T2DM-hCSCs were treated or untreated with $D+Q$ in growth media as above. Then cells were placed in bacteriological dishes and the differentiation assay was performed as above described.

\section{Statistical analysis}

All data are presented in mean \pm standard deviation. Data were analysed using t-test comparisons in GraphPad Prism version 8.0.0 for Windows, GraphPad Software, San Diego, California USA, www.graphpad.com. Differences of $p<0,05$ are considered statistically significant.

\section{Results}

\section{Increased Oxidative Stress in Diabetic Ischemic Cardiomyopathy}

Diabetes is characterized by an enhanced oxygen toxicity [26]. ROS is the distal signal of the cascade of events triggered by Diabetes that leads to the initiation of the cell death pathway in the heart [27].

To assess the effects of Diabetes on ROS-mediated cytoplasmic and DNA damage, we obtained left ventricles (LV) samples from patients with Type 2 diabetes mellitus (T2DM) and NON-diabetic mellitus (NDM) patients with post-infarct cardiomyopathy undergoing surgical coronary revascularization. As inclusion criteria an age between 50-64 years was required in order to try separate the effects of aging from Diabetes on senescence. On this premise, we included 10 T2DM and 6 NDM patients from which peri-infarct/border zone biopsies were obtained (Table 1). These samples were analyzed for the presence 
of 8-OH-deoxyguanosine (8-OH-dG), Nitrotyrosine (3-NT), and 4-hydroxinonenal (4-HNE). Diabetes status resulted in an increase of myocardial cells, which stained positive for these markers of oxidative stress when compared to NDM patients (Figure 1A-C). In particular, a higher percentage of both cardiomyocytes and CSC-enriched c-kit ${ }^{\text {pos }} \mathrm{CD} 45^{\text {neg }} \mathrm{CD} 31^{\text {neg }}$ cardiac cells positive for 8-OH-dG and 3-NT were detected in T2DM as compared with NDM (Figure 2A,B). These data show that cardiac damage in Diabetes is associated with a higher ROS production that targets both muscle cells as well as progenitor cells.

\section{Increased Oxidative Stress Associated with Increased Expression of Senescent Markers in hCSCs from Diabetic Ischemic Cardiomyopathy}

Cellular senescence is a cell state triggered by stressful insults and certain physiological processes, characterized by a prolonged and generally irreversible cell-cycle arrest with secretory

features, macromolecular damage, and altered metabolism. These mechanisms are generally interdependent as indeed oxygen toxicity and DNA damage alter telomeres, resulting in telomere shortening, cellular senescence, and cell dysfunction and/or death [28]. Although a link to organismal aging is clear, aging and senescence are not synonymous as, indeed, cells can undergo senescence, regardless of organismal age $[29,30]$. On the other hand, aging tissues experience a progressive decline in homeostatic and regenerative capacities, which has been attributed to senescence of their tissue-specific stem cells [31]. On this premise, to assess whether the exaggerated ROS in Diabetes associated with senescence of tissue-resident CSCs, independently of age, we first assessed the expression of p16 $6^{\text {INK4a }}$ in CSCs-enriched $\mathrm{c}-\mathrm{kit}^{\text {pos }} \mathrm{CD} 45^{\text {neg }} \mathrm{CD} 31^{\text {neg }}$ cardiac cells in the myocardial sections from samples obtained from T2DM patients with ischemic cardiomyopathy as compared to NDM patients. p16 ${ }^{\text {INK4a }}$ inactivates CDK4 and CDK6, which regulate cell-cycle progression in $\mathrm{G} 1$; $\mathrm{p} 16^{\mathrm{INK} 4 \mathrm{a}}$ forms binary inhibitor-CDK complexes that are highly stable and cannot be dissociated by cyclins, and cells arrest permanently [32]. Therefore, p $16^{\mathrm{INK} 4 \mathrm{a}}$ is widely used as senescent biomarker, despite not being sufficient on its own to define/detect senescent cells [33-36].

The expression of $\mathrm{p} 16^{\mathrm{INK} 4 \mathrm{a}}$ in CSCs-enriched c-kit ${ }^{\text {pos }} \mathrm{CD} 45^{\text {neg }} \mathrm{CD} 31^{\text {neg }}$ cardiac cells markedly increased in myocardial samples from T2DM ischemic cardiomyopathy when compared to NDM counterparts (Figure 2C), confirming that a higher oxidative stress couples with the induction of senescence in the cardiac progenitor pool.

To further assess senescence in the adult progenitor pool, we established cultures of human adult cardiac stem cells (hCSCs) from atrial specimens of T2DM $(n=6)$ and NDM $(n=6)$ patients undergoing to cardiac surgery (Table 1) using magnetic activated cell sorting (MACS) for CD45/CD31 negative sorting followed by c-kit positive sorting to isolate CSC-enriched c-kit ${ }^{\text {pos }} C D 45^{\text {neg }} C D 31^{\text {neg }}$ cardiac cells $[12,15,37]$. By flow cytometry, the c-kit ${ }^{\text {pos }} C D 45^{\text {neg }} C D 31^{\text {neg }}$ cardiac cells from T2DM (hereafter T2DM-hCSCs) showed a membrane phenotype typical of cardiac cells enriched with multipotent hCSCs (Figure 3A) as we have previously shown [12], with no significant difference as compared to c-kit ${ }^{\text {pos }} \mathrm{CD} 45^{\text {neg }} \mathrm{CD} 31^{\text {neg }}$ cardiac cells from NDM donors (hereafter NDM-hCSCs) (data not shown). Indeed, the hCSC-enriched c- 
kit $^{\text {pos }} C D 45^{\text {neg }} C D 31^{\text {neg }}$ cardiac cells at 14 days in culture after their primary harvest, expressed PDGFRa, CD166, CD105 and CD90, confirming the enrichment for the phenotypical identity of bona fide CSCs (Figure 3A) [37,38].

As first assessment of replicative senescence in the isolate cells, we measured telomerase activity and telomeric length (Figure 3B,C). T2DM-hCSCs showed a significant reduced telomerase activity which associated with a decreased average telomere length when compared to NDM-hCSCs (Figure 3B,C). These data suggest that Diabetes determines exaggerated oxidative stress in hCSCs that is linked to replicative senescence as demonstrated by the appearance of $\mathrm{p} 16^{\mathrm{INK} 4 \mathrm{a}}$ positive cells and reduced telomerase activity inducing telomere shortening.

\section{CSCs from non-aged diabetic subjects show impaired cell growth and myogenic differentiation potential in vitro}

One common feature of senescent cells is an essentially irreversible cell-cycle arrest that can be an alarm response instigated by deleterious stimuli or aberrant proliferation [30]. The first molecular feature associated with senescence is telomere shortening, a result of the DNA end-replication problem, during serial passages $[28,39]$. On the basis of the immunohistochemistry data showing increased p $16^{\text {INK4a }}$ expression in myocardial sections and of the in vitro data demonstrating telomerase deficit with telomere length attrition, we evaluate replicative competence of T2DM-hCSCs. To this aim, freshly-isolated T2DMhCSCs and NDM-hCSCs were plated for a week and then compared for their growth potential in vitro. T2DM-hCSCs show a significant decreased proliferation in vitro when compared to NDM-hCSCs, either assessed by growth curve kinetics over time as well as BrdU incorporation over 24 hours (Figure 4A,B). To assess whether the proliferation defect in the expansion capacity of T2DM-hCSCs was coupled with a deficit in other 'stemness' capabilities, we tested these cells for their clonal amplification by depositing half-cell per well in a 96 wells plate and for their spheroid potential by plating them in bacteriological dishes [12]. Remarkably, at 14 days after cell deposition/plating in clonogenic or spherogenic medium, respectively, clonal efficiency was 2.5-fold lower and spherogenesis 2.5-fold lower in T2DM-hCSCs when compared to NDM-hCSCs (Figure 4C,D).

Furthermore, cultured T2DM-hCSCs, expressed biomarkers of cellular senescence at significantly higher levels than NDM-hCSCs. Indeed, T2DM-hCSCs had 10-times higher levels of p16 ${ }^{\text {INK4a }}$ when compared to NDM-hCSCs (Figure 4E). Cytospin and immunocytochemistry at single cell level show that the number of p16 ${ }^{\text {INK4a }}$ positive cells within c-kit ${ }^{\text {pos }} C D 45^{\text {neg }}$ CD 3 $1^{\text {neg }}$ T2DM-hCSCs (Figure 4F) was significantly higher when compared to NDM-hCSCs (Figure 4G). Concurrently, T2DM-hCSCs show increased senescenceassociated beta-galactosidase (b-Gal) positive cells in vitro (Figure 4H). Furthermore, g-H2AX expression revealed that T2DM-hCSCs accumulate significantly more DNA damage than NDM-hCSCs in vitro (Figure 4I). Finally, T2DM-hCSCs had a significantly higher apoptotic rate when compared to NDM-hCSCs (Figure $4 J)$. 
The above data show that Diabetes increases senescence of hCSCs affecting their expansion, clonal and spheroid potential; we then tested the differentiation potential of these cells as it has been shown in different contexts that senescence and Diabetes both affect differentiation of tissue-dependent stem/progenitor cells [19-21]. Considering that myogenic differentiation is key to myocardial regeneration from endogenous progenitor cells, we evaluated cardiomyocyte differentiation of T2DM-hCSCs vs. NDMhCSCs when grown in myogenic differentiation medium using the sphere-based myogenic assay that we previously established [38]. RT-PCR data show that T2DM-hCSCs upregulated significantly less than NDM-hCSCs the main cardiac transcription factors (GATA-4, NKX2.5 and MEF2C) and myocyte contractile genes (TNNT2, ACTC1, MYH6 and MYH7) during myogenic differentiation induction (Figure 4K). Only $24 \pm 4 \%$ of T2DM-hCSCs, as compared to $52 \pm 11 \%$ of NDM-hCSCs, acquired the prototypical myocyte contractile marker cTnl by 4 weeks in culture, overall revealing a decreased capacity for myogenic differentiation (Figure 4L).

Overall, these data show that Diabetes Mellitus hampers human CSC biology, inducing a variety of hallmarks of senescence that likely contribute to the deficit of their regenerative potential.

\section{T2DM-hCSCs exhibit a defined senescence-associated secretory phenotype (SASP)}

Senescent cells secrete a plethora of factors, including pro-inflammatory cytokines and chemokines, growth modulators, angiogenic factors, and matrix metalloproteinases (MMPs), collectively known as the senescent associated secretory phenotype (SASP) [8,39]. The SASP constitutes a hallmark of senescent cells and mediates many of their patho-physiological effects, such as reinforcing and spreading senescence in autocrine and paracrine fashions [40], activating immune responses [41], hampering tissue plasticity [42] and contributing to persistent chronic inflammation (known as inflammaging) [43]. Thus, the SASP explain some of the deleterious, pro-aging effects of senescent cells. On the other hand, all these patho-physiological mechanisms are equally active in Diabetes. Therefore, we evaluated whether increased senescence markers and functional defect associated also with SASP in T2DM-hCSCs.

To this aim, we obtained culture media from T2DM-hCSCs and NDM-hCSCs after 24 hours in serum-free conditions to evaluate secretion of several SASP factors, including MMP-3, PAI1, IL-6, IL-8, IL-1 $\beta$ and GMCSF (Figure 5A,B). Interestingly, T2DM-hCSCs secreted respectively 10-, 7-, 18-, 54- and 56-fold higher amounts of IL-1A, IL-1 $\beta$, IL-6, MCP-1 and IL-8, when compared to NDM-hCSCs (Figure 5A). Concurrently, mRNA levels of MCP1, IGFBP5, MMP3, IL-6, CCL-11, IL-8, GM-CSF and PAI1 SASP factors were all increased in T2DM-hCSCs vs. NDM-hCSCs (Figure 5B).

We then treated for 7 days NDM-hCSCs with conditioned medium (CM) derived from T2DM-hCSCs (T2DM-CM) and measured cell proliferation and senescence of the treated NDM-hCSCs. As controls, NDM-hCSCs were treated either with normal growth media or CM from parallel cultures of NDM-hCSCs. Treatment of NDM-hCSCs with T2DM-CM resulted in a decreased proliferation $(p<0.05)$ (Figure 5C,D) and an increased proportion of senescent $p 16^{\text {INK4a }}$ positive $(p<0.05)$, SA- $\beta$-gal-positive and $\gamma-H 2 A X-$ 
positive hCSCs when compared to NDM-hCSCs in unconditioned medium (UM) or CM from NDM-hCSCs (Figure 5E).

These data show for the first time that Diabetes induces SASP in hCSCs isolated from non-aged patients. SASP appears to negatively affect neighbor hCSCs whereby senescence begets senescence, suggesting that targeting senescent cells could improve CSC dysfunction in Diabetes.

\section{Senolytics rescue the regenerative deficit of diabetic hCSCs}

The above data show that T2DM-hCSCs harbor a high fraction of senescent cells that produce and secrete a detrimental SASP. Because senescent cells contribute to the outcome of a variety of cardiac disease, including age-related and -unrelated cardiac diseases like anthracycline cardiotoxicity [5,44-46], much effort has been recently made to therapeutically target detrimental effects of cellular senescence. In regard with the latter, senolytic drugs are agents that selectively induce apoptosis of senescent cells by overriding anti-apoptotic pathways in senescent cells [5]. One of the most well studied senolytics therapeutic approach is the combination of Dasatinib (D) and Quercetin (Q) [5], which we show can target senescent CSCs and improve cardiac function in cardiac aging in mice [5].

To that end, T2DM-hCSCs were plated in 24-well dishes at $40 \%$ confluence and left for 2 days. Then, cells were administered with a combination of $D+Q$ at a dose of $0.25 \mu \mathrm{M} D$ with $10 \mu \mathrm{M} \mathrm{Q}$. Six hours later, $D+Q$ conditioned medium was replaced with complete fresh medium. Untreated cells served as controls. Two days later, $D+Q$ treatment was repeated as above. Two days after, T2DM-hCSCs were then analyzed for proliferation and markers of senescence, $16^{\text {INK4A }}, S A-\beta$-gal and $y-H 2 A X$. As shown in Figure $6, D+Q$ treatment removed from the cell culture the typical enlarged and flattened cell morphology of senescent cells, consistent with the senolytics combination targeting and removing senescent cells (Figure 6A). Concurrently, D+Q increased T2DM-hCSCs proliferation as evaluated by growth curve kinetics when compared to untreated control T2DM-hCSCs (Figure 6B). Accordingly, D+Q significantly reduced the number of SA- $\beta$-gal and $\gamma$-H2AX positive T2DM-hCSCs as well as the number of $p 16^{\text {INK4a }}$ senescent cells, which almost disappeared when compared to untreated T2DM-hCSCs (Figure 6C-E). Furthermore, we determined whether $D+Q$ would abrogate the SASP in T2DM-hCSCs. We found that the level of SASP factors secreted by T2DM-hCSCs was significantly reduced by the administration of $D+Q$ combination (Figure 6F).

Finally, we checked whether $D+Q$ pre-treatment was able to rescue the altered myogenesis potential of diabetic hCSCs. To that end, T2DM-hCSCs were treated or untreated D+Q as above in growth media and then cells were plated in bacteriological dishes for sphere formation [12]. CSC-derived cardiospheres were then plated in laminin for additional 7 days in the stage specific cardiomyogenic media [12]. Interestingly, we found that D+Q pre-treatment restored the myogenic capacity of T2DM-hCSCs as indeed this senolytic combination significantly increased myogenic transcription factor and myogenic contractile gene 
expression as compared to untreated cells in vitro (Figure 6G). Accordingly, $D+Q$ increased the number of T2DM-hCSC-derived cTnl ${ }^{\text {pos }}$ cardiomyocytes after 14 days in differentiation media (Figure $6 \mathrm{H}$ ).

Overall, these findings document that clearance of senescent cells using a combination of $D+Q$ senolytics abrogates the SASP and restores a fully proliferative- and differentiation- competent hCSCs pool in T2DM.

\section{Discussion}

The main findings emanating from this study are that: i) Ischemic damaged myocardial tissue of T2DM patients is characterized by an exaggerated oxidative stress targeting both cardiomyocytes and cardiac stem/progenitor cells (CSCs); ii) Increased oxidative stress in the myocardium of non-aged T2DM patients associates with an increased number of senescent and dysfucntional T2DM-hCSCs as shown by increased $\mathrm{p} 16^{\mathrm{INK} 4 \mathrm{a}}$ expression, reduced telomerase activity and telomere length, reduced proliferation, clonogenesis/spherogenesis and myogenic differentiation; iii) T2DM-hCSCs from non-aged subjects show a senescence-associated secretory phenotype (SASP), as demonstrated by the increased secretion of several SASP factors, including MMP-3, PAI1, IL-6, IL-8, IL-1 $\beta$ and GM-CSF; iv) a combination of two senolytics, Dasatinib and Quercetin, clear senescent T2DM-hCSCs restoring expansion and myogenic differentiation capacities of the diabetic hCSC pool.

T2DM is a pre-conditioning and powerful driver of organismal aging [47]. The biological foundations of aging primarily involve cellular senescence and T2DM is plethoric in senescence-driving factors [47]. An intense debate has existed so far addressing whether senescence precedes or follows the onset of perpetual inflammation and insulin resistance (IR) in T2DM [48]. Independently from "who-precedes-who," diabetic patients experience an obvious accelerated aging process that increases their susceptibility to morbidity and earlier mortality [49]. Hence, diabetes-affected patients have a significantly shorter life expectancy than non-diabetic individuals, and cardiovascular disease accounts for a large part of the excess mortality [50]. Indeed, adults with T2DM have 2-4 times increased cardiovascular risk compared with adults without diabetes, and the risk rises with worsening glycemic control (T2DM) [1].

Adult tissue-specific stem cells are multipotent cells that are considered a lifelong cellular reservoir to ensure the continuous generation, replacement, and restitution of multiple tissue lineages [51, 52]. Therefore, adult stem cells play a vital role in preventing the aging of organs and tissues, and can delay aging. However, during aging, these cells also undergo some detrimental changes such as alterations in the microenvironment, a decline in the regenerative capacity, and loss of function so that aging has been linked to tissue-specific adult stem cell exhaustion [31]. Converging evidence conclusively demonstrated how toxic high glucose load may be for survival, differentiation plasticity, and regenerative competence for different stem cells lineages [47, 53-57]. The self-sustaining vicious circle amalgamated by hyperglycemia/mitochondrial dysfunction/oxidative stress appears as a master driver to adult mesenchymal stem cell senescence [58]. Accordingly, the diabetic pro-oxidative environment is a major contributing factor for premature adult stem cell senescence and functional deficit [47, 58-62]. Therefore, 
Aging and T2DM drive adult stem cell senescence and regenerative deficit and conversely adult stem cell senescence and regenerative deficit drive the progressive pathology in aging and diabetes.

Like other tissue specific adult stem/progenitor cells, also CSCs are not immortal [16]. They undergo cellular senescence characterized by increased ROS production and oxidative stress and loss of telomere/telomerase integrity in response to a variety of physiological and pathological demands with aging [16]. Nevertheless, the old myocardium preserves an endogenous functionally competent CSC cohort which appears to be resistant to the senescent phenotype occurring with aging [16]. The latter envisions the phenomenon of CSC ageing as a result of a stochastic and therefore reversible cell autonomous process, whereby targeting the senescent cells would benefit the recovery of a healthy CSC cohort [16]. Concurrently, T2DM impairs the in vitro proliferative and differentiation potential of human cardiac stem/progenitor cells, worsening their senescence phenotype when compared with CSCs from non-diabetic ischemic patients [22]. Additionally, miR-34a is significantly upregulated while SIRT1 is downregulated in adult CSCs harvested from T2DM patients, which is associated with a higher proapoptotic caspase-3/7 activity [63]. All the above studies were, however, conducted in hCSCs isolated from diabetic old patients ( $>65$ years), which could not clearly distinguish the role of age from DM on hCSC function. On the other hand, in an animal model of insulin-dependent DM in young mice, the myocardial accumulation of ROS drives CSC senescence through the expression of p53 and p16 INK4a proteins and telomere erosion [27]. p66 ${ }^{\text {shc }}$ knockout inhibits CSC senescence and death, preventing the senescent phenotype and the

development of cardiac failure by T2DM [27]. Additionally, Diabetes persistently decreases the ability of isolated CSCs from young male mice to proliferate, survive oxidative insults, and differentiate, which can be explained at least in part by an uncoupling of biosynthetic glucose metabolism pathways [64]. Diabetes suppresses CSC activation through a diminished SCF expression in the heart following permanent LAD ligation in young/adult rats [20]. Altogether, these data postulate the tantalizing hypothesis that the premature cellular senescence and ageing of resident CSCs underpins the development of diabetic heart disease [10].

In the present study we show premature aging in hCSCs isolated from non-aged ( $<65$ years old) patients with T2DM. Indeed, T2DM hCSCs harbor a significant fraction of senescent cells, with exaggerated ROS, DNA damage and telomere erosion. In particular, we provide evidence that independently from age, T2DM-hCSCs have a defined pathologic SASP, which can drive senescence into healthy hCSCs to senescence. Furthermore, T2DM-hCSCs have a reduced capacity to differentiate into cardiomyocytes in vitro. The premature senescence is clearly central to the regenerative defect imposed by Diabetes on hCSCs as demonstrated by the elimination of senescent cells by senolytic treatment, a combination of Dasatinib and Quercetin, which rescued the expansion and myogenic capacity of T2DM-hCSCs. Therefore, this data suggests that eliminating senescent hCSCs by senolytic therapy in vivo could be a therapeutic strategy to prevent hCSC dysfunction even at early stages of Diabetic disease. 
Although hampered by the difficulty and limitation of working with samples of human tissue, which makes the data presented here mainly descriptive and falling short of being able to establish direct cause-effect relationship(s) (as it is also the case for most work based on primary human tissue), our findings strongly point to Diabetes hampering human CSC biology, inducing a variety of hallmarks of senescence in non-aged subjects that contribute to the deficit of their regenerative potential. Diabetes induces a pathologic SASP in hCSCs that spread to otherwise healthy hCSCs, reducing the functional pool of hCSCs. This conclusion is reinforced by the evidence that clearance of senescent cells by senolytics abrogates the SASP and restores a fully proliferative- and differentiation- competent hCSC pool in T2DM. Nevertheless, this study cannot fully extrapolate the role of ischemic damage to the observed effects of Diabetes on hCSCs in vivo. Indeed, despite included patients were homogenous for number of vessel diseases and infarct area, Diabetes is known to determine more intense microvascular ischemia, which may have affected the status and function of hCSCs. On the other hand, isolated hCSCs were obtained from right atrial tissue which were not ischemic and therefore most likely the observed effects of Diabetes is independent form ischemia at least for the in vitro data. Yet, further work is needed to fully clarify this issue. Finally, the data of this study in human cells in vitro surely improve our understanding of the cellular mechanisms behind CSC dysfunction in T2DM, identifying senescent cells as potential drug targets and guiding the design of clinical test of candidate $D+Q$ for the myocardial regenerative defect of the diabetic heart. However, human cells in vitro cannot fully recapitulate the human scenario whereby human organoids, stem cell-derived 3D culture systems, re-create the architecture and physiology of human organs in remarkable detail, providing unique opportunities for the study of human disease [65]. It will be therefore truly interesting to further evaluate the role of premature senescence of hCSCs in the context of diabetic human cardiac organoids in the next future [66].

\section{Conclusions}

DM hampers human CSC biology, inducing cellular senescence and senescence associate secretory phenotype (SASP) in non-aged subjects (<65 years) that contribute to a significant deficit of their regenerative potential and in particular to their ability to differentiate in new cardiomyocytes. Clearance of senescent cells by senolytics abrogates the SASP and restores a fully proliferative- and differentiationcompetent hCSC pool in T2DM. Therefore, senolytics may represent a potential therapeutic approach to prevent or treat the reduced regenerative potential of the diabetic heart.

\section{Declarations}

Ethics approval and consent to participate: Ethics approval and consent to participate are both stated in the METHODS section above.

Consent for publication: Not Applicable.

Availability of data and materials: All data generated or analysed during this study are included in this published article 
Competing interests: "The authors declare no conflict of interest."

Funding: This research was funded by Grants from the Ministry of University and Research PRIN2015 2015ZTT5KB_004; PRIN2017NKB2N4_005; PON-AIM - 1829805-2

Authors' contributions: Conceptualization: D.T., K.U. and E.C.; Design of the work: A.DeA., G.M.E-H.,K.U., D.T., and E.C. Data Acquisition and Analysis: F.M., M.S., L.S., D.C., C.M., N.S. M.G.; Interpretation: D.F., M.T., F.C.S, P.M., A.DeA., K.U., D.T., and E.C. Writing original draft preparation and editing F.M., G.M.E-H., M.R., E.C., K.U., D.T.. All authors have read and agreed to the published version of the manuscript.

Acknowledgments: Nothing to acknowledge

\section{References}

1. Dunlay SM, Givertz MM, Aguilar D, Allen LA, Chan M, Desai AS, Deswal A, Vaughan Dickson V, Kosiborod MN, Lekavich CL, McCoy RG, Mentz RJ, Piña IL. Type 2 Diabetes Mellitus and Heart Failure: A Scientific Statement From the American Heart Association and the Heart Failure Society of America. American Heart Association Heart Failure and Transplantation Committee of the Council on Clinical Cardiology; Council on Cardiovascular and Stroke Nursing and the Heart Failure Society of America. This statement does not represent an update of the 2017 ACC/AHA/HFSA heart failure guideline update. Circulation. 2019 Aug 13;140(7):e294-e324.

2. Gude NA, Broughton KM, Firouzi F, Sussman MA. Cardiac ageing: extrinsic and intrinsic factors in cellular renewal and senescence. Nat Rev Cardiol. 2018 Sep;15(9):523-542.

3. Halim M, Halim A. The effects of inflammation, aging and oxidative stress on the pathogenesis of diabetes mellitus (type 2 diabetes). Diabetes Metab Syndr. 2019;13(2):1165-1172.

4. van Deursen JM. The role of senescent cells in ageing. Nature. 2014 May 22; 509(7501):439-46.

5. Lewis-McDougall FC, Ruchaya PJ, Domenjo-Vila E, Shin Teoh T, Prata L, Cottle BJ, Clark JE, Punjabi PP, Awad W, Torella D, Tchkonia T, Kirkland JL, Ellison-Hughes GM. Aged-senescent cells contribute to impaired heart regeneration. Aging Cell. 2019 Jun;18(3):e12931.

6. Tchkonia, T, Zhu Y, van Deursen J, Campisi J, Kirkland JL. Cellular senescence and the senescent secretory phenotype: Therapeutic opportunities. The Journal of Clinical Investigation. 2013. 123(3), 966-972.

7. Xu M, Palmer AK, Ding H, Weivoda MM, Pirtskhalava T, White TA, Sepe A, Johnson KO, Stout MB, Giorgadze N, Jensen MD, LeBrasseur NK, Tchkonia T, Kirkland JL. Targeting senescent cells enhances adipogenesis and metabolic function in old age. Elife. 2015 Dec 19;4:e12997.

8. Acosta JC, Banito A, Wuestefeld T, Georgilis A, Janich P, Morton JP, Athineos D, Kang TW, Lasitschka F, Andrulis M, Pascual G, Morris KJ, Khan S, Jin H, Dharmalingam G, Snijders AP, Carroll T, Capper D, Pritchard C, Inman GJ, Longerich T, Sansom OJ, Benitah SA, Zender L, Gil J. A complex secretory program orchestrated by the inflammasome controls paracrine senescence. Nat Cell Biol. 2013 Aug;15(8):978-90. 
9. Shakeri H, Lemmens K, Gevaert AB, De Meyer GRY, Segers VFM. Cellular senescence links aging and diabetes in cardiovascular disease. Am J Physiol Heart Circ Physiol. 2018 Sep 1;315(3):H448-H462.

10. Cianflone E, Torella M, Biamonte F, De Angelis A, Urbanek K, Costanzo FS, Rota M, Ellison-Hughes GM, Torella D. Targeting Cardiac Stem Cell Senescence to Treat Cardiac Aging and Disease. Cells. 2020 Jun 26;9(6):1558.

11. Cianflone E, Aquila I, Scalise M, Marotta P, Torella M, Nadal-Ginard B, Torella D. Molecular basis of functional myogenic specification of Bona Fide multipotent adult cardiac stem cells. Cell Cycle. 2018;17(8):927-946.

12. Scalise M, Torella M, Marino F, Ravo M, Giurato G, Vicinanza C, Cianflone E, Mancuso T, Aquila I, Salerno L, Nassa G, Agosti V, De Angelis A, Urbanek K, Berrino L, Veltri P, Paolino D, Mastroroberto P, De Feo M, Viglietto G, Weisz A, Nadal-Ginard B, Ellison-Hughes GM, Torella D. Atrial myxomas arise from multipotent cardiac stem cells. Eur Heart J. 2020 Dec 1;41(45):4332-4345.

13. Scalise M, Marino F, Cianflone E, Mancuso T, Marotta P, Aquila I, Torella M, Nadal-Ginard B, Torella D. Heterogeneity of Adult Cardiac Stem Cells. Adv Exp Med Biol. 2019;1169:141-178.

14. Di Siena S, Gimmelli R, Nori SL, Barbagallo F, Campolo F, Dolci S, Rossi P, Venneri MA, Giannetta E, Gianfrilli D, Feigenbaum L, Lenzi A, Naro F, Cianflone E, Mancuso T, Torella D, Isidori AM, Pellegrini M. Activated c-Kit receptor in the heart promotes cardiac repair and regeneration after injury. Cell Death Dis. 2016 Jul 28;7(7):e2317.

15. Vicinanza C, Aquila I, Cianflone E, Scalise M, Marino F, Mancuso T, Fumagalli F, Giovannone ED, Cristiano F, laccino E, Marotta P, Torella A, Latini R, Agosti V, Veltri P, Urbanek K, Isidori AM, Saur D, Indolfi C, Nadal-Ginard B, Torella D. Kit ${ }^{\text {cre }}$ knock-in mice fail to fate-map cardiac stem cells. Nature. 2018 Mar 21;555(7697):E1-E5.

16. Cianflone E, Torella M, Chimenti C, De Angelis A, Beltrami AP, Urbanek K, Rota M, Torella D. Adult Cardiac Stem Cell Aging: A Reversible Stochastic Phenomenon? Oxid Med Cell Longev. 2019 Feb 7;2019:5813147.

17. Marino F, Scalise M, Cianflone E, Mancuso T, Aquila I, Agosti V, Torella M, Paolino D, Mollace V, NadalGinard B, Torella D. Role of c-Kit in Myocardial Regeneration and Aging. Front Endocrinol (Lausanne). 2019 Jun 19;10:371.

18. Tchkonia, T, Kirkland JL. Aging, cell senescence, and chronic disease: Emerging therapeutic strategies. JAMA. 2018;320(13), 1319-1320.

19. Zhang X, Meng K, Pu Y, Wang C, Chen Y, Wang L. Hyperglycemia Altered the Fate of Cardiac Stem Cells to Adipogenesis through Inhibiting the $\beta$-Catenin/TCF-4 Pathway. Cell Physiol Biochem. 2018;49(6):2254-2263.

20. She T, Wang X, Gan Y, Kuang D, Yue J, Ni J, Zhao X, Wang G. Hyperglycemia suppresses cardiac stem cell homing to peri-infarcted myocardium via regulation of ERK1/2 and p38 MAPK activities. Int J Mol Med. 2012 Dec;30(6):1313-20.

21. Molgat AS, Tilokee EL, Rafatian G, Vulesevic B, Ruel M, Milne R, Suuronen EJ, Davis DR. Hyperglycemia inhibits cardiac stem cell-mediated cardiac repair and angiogenic capacity. 
Circulation. 2014 Sep 9;130(11 Suppl 1):S70-6.

22. Vecellio M, Spallotta F, Nanni S, Colussi C, Cencioni C, Derlet A, Bassetti B, Tilenni M, Carena MC, Farsetti A, Sbardella G, Castellano S, Mai A, Martelli F, Pompilio G, Capogrossi MC, Rossini A, Dimmeler S, Zeiher A, Gaetano C. The histone acetylase activator pentadecylidenemalonate 1b rescues proliferation and differentiation in the human cardiac mesenchymal cells of type 2 diabetic patients. Diabetes. 2014 Jun;63(6):2132-47.

23. Debacq-Chainiaux F, Erusalimsky JD, Campisi J, Toussaint O. Protocols to detect senescenceassociated beta-galactosidase (SA-beta-gal) activity, a biomarker of senescent cells in culture and in vivo. Nat Protoc. 2009;4(12):1798-806.

24. Xin H. Telomeric repeat amplification protocol: measuring the activity of the telomerase. Methods Mol Biol. 2011;735:107-11.

25. Accattato F, Greco M, Pullano SA, Carè I, Fiorillo AS, Pujia A, Montalcini T et al. Effects of acute physical exercise on oxidative stress and inflammatory status in young, sedentary obese subjects. PLoS One. 2017; 12(6): e0178900.

26. Pham-Huy LA, He H, Pham-Huy C. Free radicals, antioxidants in disease and health. Int J Biomed Sci. 2008 Jun;4(2):89-96.

27. Rota M, LeCapitaine N, Hosoda T, Boni A, De Angelis A, Padin-Iruegas ME, Esposito G, Vitale S, Urbanek K, Casarsa C, Giorgio M, Lüscher TF, Pelicci PG, Anversa P, Leri A, Kajstura J. Diabetes promotes cardiac stem cell aging and heart failure, which are prevented by deletion of the p66shc gene. Circ Res. 2006 Jul 7;99(1):42-52.

28. Coluzzi E, Colamartino M, Cozzi R, Leone S, Meneghini C, O'Callaghan N, Sgura A. Oxidative stress induces persistent telomeric DNA damage responsible for nuclear morphology change in mammalian cells. PLoS One. 2014 Oct 29;9(10):e110963.

29. Rodier F, Muñoz DP, Teachenor R, Chu V, Le O, Bhaumik D, Coppé JP, Campeau E, Beauséjour CM, Kim $\mathrm{SH}$, Davalos AR, Campisi J. DNA-SCARS: distinct nuclear structures that sustain damage-induced senescence growth arrest and inflammatory cytokine secretion. J Cell Sci. 2011 Jan 1;124(Pt 1):6881

30. Gorgoulis V, Adams PD, Alimonti A, Bennett DC, Bischof O, Bishop C, Campisi J, Collado M, Evangelou K, Ferbeyre G, Gil J, Hara E, Krizhanovsky V, Jurk D, Maier AB, Narita M, Niedernhofer L, Passos JF, Robbins PD, Schmitt CA, Sedivy J, Vougas K, von Zglinicki T, Zhou D, Serrano M, Demaria M. Cellular Senescence: Defining a Path Forward. Cell. 2019 Oct 31;179(4):813-827.

31. Oh J, Lee YD, Wagers AJ. Stem cell aging: mechanisms, regulators and therapeutic opportunities. Nat Med. 2014 Aug;20(8):870-80.

32. McConnell BB, Gregory FJ, Stott FJ, Hara E, Peters G. Induced expression of p16(INK4a) inhibits both CDK4- and CDK2-associated kinase activity by reassortment of cyclin-CDK-inhibitor complexes. Mol Cell Biol. 1999 Mar;19(3):1981-9.

33. Jeyapalan JC, Sedivy JM. Cellular senescence and organismal aging. Mechanisms of Ageing and Development. 2008; 129(7), 467-474. 
34. Kirkland JL, Hollenberg $\mathrm{CH}$, Gillon WS. Age, anatomic site, and the replication and differentiation of adipocyte precursors. American Journal of Physiology. 1990;258(2 Pt 1), C206-C210

35. Sharpless NE, DePinho RA. How stem cells age and why this makes us grow old. Nature Reviews Molecular Cell Biology. 2007;8, 703.

36. Liu JY, Souroullas GP, Diekman BO, Krishnamurthy J, Hall BM, Sorrentino JA, Parker JS, Sessions GA, Gudkov AV, Sharpless NE. Cells exhibiting strong p16INK4a promoter activation in vivo display features of senescence. Proc Natl Acad Sci U S A. 2019 Feb 12;116(7):2603-2611.

37. Vicinanza C, Aquila I, Scalise M, Cristiano F, Marino F, Cianflone E, Mancuso T, Marotta P, Sacco W, Lewis FC, Couch L, Shone V, Gritti G, Torella A, Smith AJ, Terracciano CM, Britti D, Veltri P, Indolfi C, Nadal-Ginard B, Ellison-Hughes GM, Torella D. Adult cardiac stem cells are multipotent and robustly myogenic: c-kit expression is necessary but not sufficient for their identification. Cell Death Differ. 2017 Dec;24(12):2101-2116.

38. Smith AJ, Lewis FC, Aquila I, Waring CD, Nocera A, Agosti V, Nadal-Ginard B, Torella D, Ellison GM. Isolation and characterization of resident endogenous c-Kit+ cardiac stem cells from the adult mouse and rat heart. Nat Protoc. 2014 Jul;9(7):1662-81.

39. Kumari R, Jat P. Mechanisms of Cellular Senescence: Cell Cycle Arrest and Senescence Associated Secretory Phenotype. Front Cell Dev Biol. 2021 Mar 29;9:645593.

40. Hoare M, Narita M. Transmitting senescence to the cell neighbourhood. Nat Cell Biol. 2013 Aug;15(8):887-9.

41. Burton DGA, Stolzing A. Cellular senescence: Immunosurveillance and future immunotherapy. Ageing Res Rev. 2018 May;43:17-25.

42. Ritschka B, Storer M, Mas A, Heinzmann F, Ortells MC, Morton JP, Sansom OJ, Zender L, Keyes WM. The senescence-associated secretory phenotype induces cellular plasticity and tissue regeneration. Genes Dev. 2017 Jan 15;31(2):172-183.

43. Franceschi C, Garagnani P, Vitale G, Capri M, Salvioli S. Inflammaging and 'Garb-aging'. Trends Endocrinol Metab. 2017 Mar;28(3):199-212.

44. Carresi C, Musolino V, Gliozzi M, Maiuolo J, Mollace R, Nucera S, Maretta A, Sergi D, Muscoli S, Gratteri S, Palma E, Bosco F, Giancotta C, Muscoli C, Marino F, Aquila I, Torella D, Romeo F, Mollace V. Anti-oxidant effect of bergamot polyphenolic fraction counteracts doxorubicin-induced cardiomyopathy: Role of autophagy and c-kit ${ }^{\text {pos }} \mathrm{CD} 45^{\text {neg }} \mathrm{CD} 31^{\text {neg }}$ cardiac stem cell activation. $\mathrm{J}$ Mol Cell Cardiol. 2018 Jun;119:10-18.

45. Piegari E, De Angelis A, Cappetta D, Russo R, Esposito G, Costantino S, Graiani G, Frati C, Prezioso L, Berrino L, Urbanek K, Quaini F, Rossi F. Doxorubicin induces senescence and impairs function of human cardiac progenitor cells. Basic Res Cardiol. 2013 Mar;108(2):334.

46. Cappetta D, De Angelis A, Sapio L, Prezioso L, Illiano M, Quaini F, Rossi F, Berrino L, Naviglio S, Urbanek K. Oxidative Stress and Cellular Response to Doxorubicin: A Common Factor in the Complex Milieu of Anthracycline Cardiotoxicity. Oxid Med Cell Longev. 2017;2017:1521020. 
47. Berlanga-Acosta JA, Guillén-Nieto GE, Rodríguez-Rodríguez N, Mendoza-Mari Y, Bringas-Vega ML, Berlanga-Saez JO, García Del Barco Herrera D, Martinez-Jimenez I, Hernandez-Gutierrez S, ValdésSosa PA. Cellular Senescence as the Pathogenic Hub of Diabetes-Related Wound Chronicity. Front Endocrinol (Lausanne). 2020 Sep 16;11:573032.

48. Prattichizzo F, de Nigris V, La Sala L, Procopio AD, Olivieri F, Ceriello A. "Inflammaging" as a druggable target: a senescence-associated secretory phenotype-centered view of Type 2 diabetes. Oxid Med Cell Longev. 2016; 2016:1810327.

49. Perkisas S, Vandewoude M. Where frailty meets diabetes. Diabetes Metab Res Rev. 2016 Jan;32 Suppl 1:261-7.

50. Alison K. Wright, Evangelos Kontopantelis, Richard Emsley, lain Buchan, Naveed Sattar, Martin K. Rutter, Darren M. Ashcroft. Life Expectancy and Cause-Specific Mortality in Type 2 Diabetes: A Population-Based Cohort Study Quantifying Relationships in Ethnic Subgroups. Diabetes Care Mar. 2017; 40 (3) 338-345

51. Andrzejewska A, Lukomska B, Janowski M. Concise Review: Mesenchymal Stem Cells: From Roots to Boost. Stem Cells. 2019 Jul;37(7):855-864.

52. Aquila I, Cianflone E, Scalise M, Marino F, Mancuso T, Filardo A, Smith AJ, Cappetta D, De Angelis A, Urbanek K, Isidori AM, Torella M, Agosti V, Viglietto G, Nadal-Ginard B, Ellison-Hughes GM, Torella D. c-kit Haploinsufficiency impairs adult cardiac stem cell growth, myogenicity and myocardial regeneration. Cell Death Dis. 2019 Jun 4;10(6):436.

53. Yang G, Jia Y, Li C, Cheng Q, Yue W, Pei X. Hyperglycemic stress impairs the stemness capacity of kidney stem cells in rats. PLoS ONE. 2015; 10:e0139607.

54. Dienelt A, zur Nieden NI. Hyperglycemia impairs skeletogenesis from embryonic stem cells by affecting osteoblast and osteoclast differentiation. Stem Cells Dev. 2011; 20:465-74.

55. Xu W, Yu W, Gao H, Wang L, Liu X, Wang Y. Effects of high glucose and high lipid on proliferation and apoptosis of human umbilical cord mensenchymal stem cells. Chin J Tissue Eng Res. 2012; 16:5001- 5 .

56. Krankel N, Adams V, Linke A, Gielen S, Erbs S, Lenk K, et al. Hyperglycemia reduces survival and impairs function of circulating bloodderived progenitor cells. Arterioscler Thromb Vasc Biol. 2005; 25:698- 703.

57. Torella D, laconetti C, Tarallo R, Marino F, Giurato G, Veneziano C, Aquila I, Scalise M, Mancuso T, Cianflone E, Valeriano C, Marotta P, Tammè L, Vicinanza C, Sasso FC, Cozzolino D, Torella M, Weisz A, Indolfi C. miRNA Regulation of the Hyperproliferative Phenotype of Vascular Smooth Muscle Cells in Diabetes. Diabetes. 2018 Dec;67(12):2554-2568.

58. Vono R, Jover Garcia E, Spinetti G, Madeddu P. Oxidative Stress in Mesenchymal Stem Cell Senescence: Regulation by Coding and Noncoding RNAs. Antioxid Redox Signal. 2018 Sep 20;29(9):864-879.

59. Cassidy FC, Shortiss C, Murphy CG, Kearns SR, Curtin W, De Buitléir C, O'Brien T, Coleman CM. Impact of Type 2 Diabetes Mellitus on Human Bone Marrow Stromal Cell Number and Phenotypic 
Characteristics. Int J Mol Sci. 2020 Apr 2;21(7):2476.

60. Ko KI, Coimbra LS, Tian C, Alblowi J, Kayal RA, Einhorn TA, Gerstenfeld LC, Pignolo RJ, Graves DT. Diabetes reduces mesenchymal stem cells in fracture healing through a TNFalpha-mediated mechanism. Diabetologia. 2015; 58:633- 42.

61. van de Vyver M, Niesler C, Myburgh KH, Ferris WF. Delayed wound healing and dysregulation of IL6/STAT3 signalling in MSCs derived from pre-diabetic obese mice. Mol Cell Endocrinol. 2016; 426:1- 10 .

62. Lu H, Wu X, Wang Z, Li L, Chen W, Yang M, Huo D, Zeng W, Zhu C. Erythropoietin-activated mesenchymal stem cells promote healing ulcers by improving microenvironment. J Surg Res. 2016; 205:464-73.

63. Fomison-Nurse I, Saw EEL, Gandhi S, Munasinghe PE, Van Hout I, Williams MJA, Galvin I, Bunton R, Davis $\mathrm{P}$, Cameron V, Katare R. Diabetes induces the activation of pro-ageing miR-34a in the heart, but has differential effects on cardiomyocytes and cardiac progenitor cells. Cell Death Differ. 2018;25:1336-1349.

64. Salabei JK, Lorkiewicz PK, Mehra P, Gibb AA, Haberzettl P, Hong KU, Wei X, Zhang X, Li Q, Wysoczynski M, Bolli R, Bhatnagar A ,Hill BG. Type 2 Diabetes Dysregulates Glucose Metabolism in Cardiac Progenitor Cells. J Biol Chem. 2016;291(26):13634-13648.

65. Kim J, Koo BK, Knoblich JA. Human organoids: model systems for human biology and medicine. Nat Rev Mol Cell Biol. 2020;21(10):571-584.

66. Nugraha B, Buono MF, von Boehmer L, Hoerstrup SP, Emmert MY. Human Cardiac Organoids for Disease Modeling. Clin Pharmacol Ther. 2019 Jan;105(1):79-85

\section{Tables}

Table 1. Characteristics of the Patients Enrolled in the Study 


\begin{tabular}{|c|c|c|c|}
\hline & $\begin{array}{l}\text { T2DM Patients } \\
n=16\end{array}$ & $\begin{array}{l}\text { NDM Patients } \\
n=12\end{array}$ & \\
\hline Sex, M/F & $14 / 2$ & $11 / 1$ & 0.54 \\
\hline Age, y & $59.3 \pm 3.8$ & $59.4 \pm 4.1$ & 0.97 \\
\hline Duration of diabetes, $\mathrm{y}$ & $7.3 \pm 4.3$ & - & \\
\hline $\mathrm{BMI}, \mathrm{kg} / \mathrm{m}^{2}$ & $27.7 \pm 3.1$ & $28.8 \pm 3.5$ & 0.39 \\
\hline $\mathrm{HbA}_{1 \mathrm{c}}, \%$ & $9.1 \pm 3.8$ & - & \\
\hline FBG, mg/dl & $166.4 \pm 64.1$ & $92.7 \pm 9.3$ & $<0.01$ \\
\hline LDL cholesterol, mg/dl & $108.6 \pm 48.3$ & $118.4 \pm 43.1$ & 0.58 \\
\hline HDL cholesterol, mg/dl & $41 \pm 15.4$ & $47.2 \pm 17.0$ & 0.33 \\
\hline $\mathrm{TG}, \mathrm{mg} / \mathrm{dl}$ & $168.9 \pm 106.5$ & $139.7 \pm 67.4$ & 0.38 \\
\hline $\mathrm{SBP}, \mathrm{mm} \mathrm{Hg}$ & $133.6 \pm 14.7$ & $131.2 \pm 19.0$ & 0.75 \\
\hline $\mathrm{DBP}, \mathrm{mm} \mathrm{Hg}$ & $79.2 \pm 9.1$ & $79.7 \pm 11.0$ & 0.91 \\
\hline Three-vessel CHD, $\mathrm{n}$ & 8 & 8 & 0.67 \\
\hline Two-vessel CHD, $\mathrm{n}$ & 5 & 4 & 0.92 \\
\hline \multicolumn{4}{|l|}{ Therapy, $\mathrm{n}$} \\
\hline Statins & 16 & 12 & \\
\hline Aspirin & 16 & 12 & \\
\hline ß-Blockers & 16 & 10 & 0.31 \\
\hline ACEIs/ARBS & 16 & 10 & 0.29 \\
\hline Nitrates & 0 & 0 & \\
\hline
\end{tabular}

Quantitative data are expressed as mean \pm SD. Binary data are reported by counts. ${ }^{*} \mathrm{P}<0.01$ vs DM patients. Comparisons of the quantitative data have been made through use of Student's t test for independent samples. The $\chi 2$ test was used to compare binary data. T2DM indicates Type 2 Diabetes Mellitus; NDM non diabetes Mellitus; BMI body mass index; FBG, fasting blood glucose; LDL, low-density lipoprotein; HDL, high-density lipoprotein; TG, triglycerides; SDP, systolic blood pressure; DBP, diastolic blood pressure; CHD, coronary heart disease; ACEI, angiotensin-converting enzyme-inhibitor; ARB, angiotensin II receptor blocker.

\section{Table 2. Flow cytometry antibodies}




\begin{tabular}{|l|l|l|l|}
\hline Antigen & Antibody ID & Company & Application \\
\hline C-kit & A3C6E2 & Miltenyi Biotec & FC \\
\hline CD184 (CXCR4) & 12G5 & Miltenyi Biotec & FC \\
\hline CD9 & SN4 C3-3A2 & Miltenyi Biotec & FC \\
\hline CD44 & DB105 & Miltenyi Biotec & FC \\
\hline CD81 & 5A6 & BioLegend & FC \\
\hline CD90 & DG3 & Miltenyi Biotec & FC \\
\hline CD166 & 3A6 & BioLegend & FC \\
\hline CD105 & 43A4E1 & Miltenyi Biotec & FC \\
\hline MDR-1 & UIC2 & BioLegend & FC \\
\hline PDGFR- $\alpha$ & 16A1 & BioLegend & FC \\
\hline CD150 & REA151 & Miltenyi Biotec & FC \\
\hline CD31 & AC128 & Miltenyi Biotec & FC \\
\hline CD45 & REA747 & Miltenyi Biotec & FC \\
\hline CD34 & AC136 & Miltenyi Biotec & FC \\
\hline Mouse IgG1 - Isotype control antibody & & Miltenyi Biotec & FC \\
\hline Mouse IgG2a - Isotype control antibody & & Miltenyi Biotec & FC \\
\hline Mouse IgG2b - Isotype control antibody & & Miltenyi Biotec & FC \\
\hline
\end{tabular}

FC denotes Flow Cytometry

Table 3. TaqMan Primers List

\begin{tabular}{|l|l|}
\hline GAPDH & Hs02786624_g1 \\
\hline MEF2C & Hs00231149_m1 \\
\hline NKX2.5 & Hs00231763_m1 \\
\hline GATA4 & Hs00171403_m1 \\
\hline MYH6 & Hs01101425_m1 \\
\hline MYH7 & Hs01110632_m1 \\
\hline TNNT2 & Hs00943911_m1 \\
\hline ACTC1 & Hs01109515_m1 \\
\hline CCL2 & Hs00234140_m1 \\
\hline CXCL8 & Hs00174103_m1 \\
\hline GM-CSF & Hs00929873_m1 \\
\hline IGF-BP & Hs00181213_m1 \\
\hline$I L-6$ & Hs00174131_m1 \\
\hline PAI-1 & Hs00167155_m1 \\
\hline MMP-3 & Hs00968305_m1 \\
\hline$C C L-11$ & Hs00237013_m1 \\
\hline
\end{tabular}

\section{Figures}


A

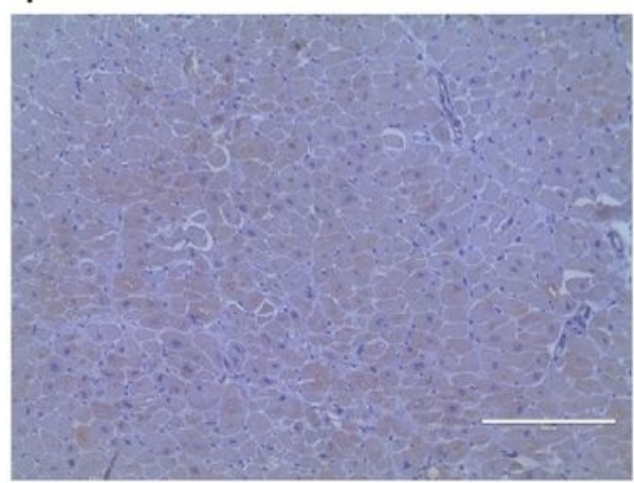

NDM

B

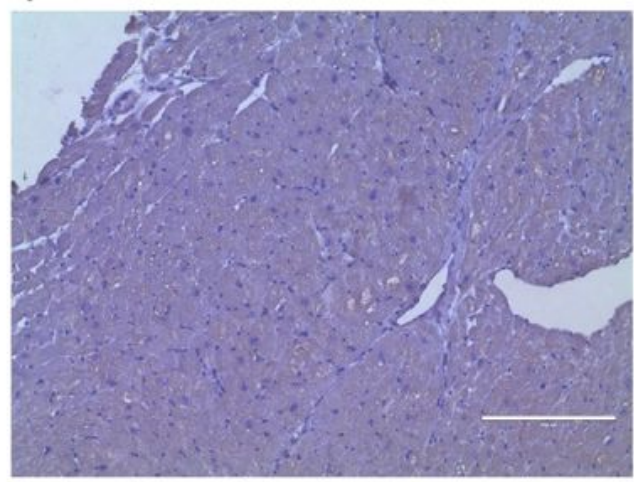

NDM

C

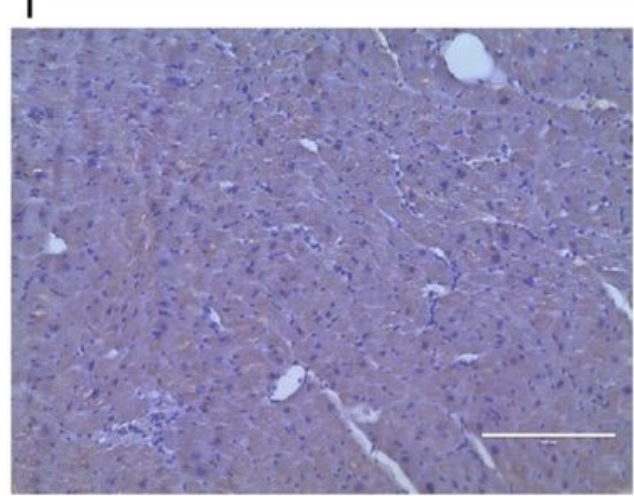

NDM

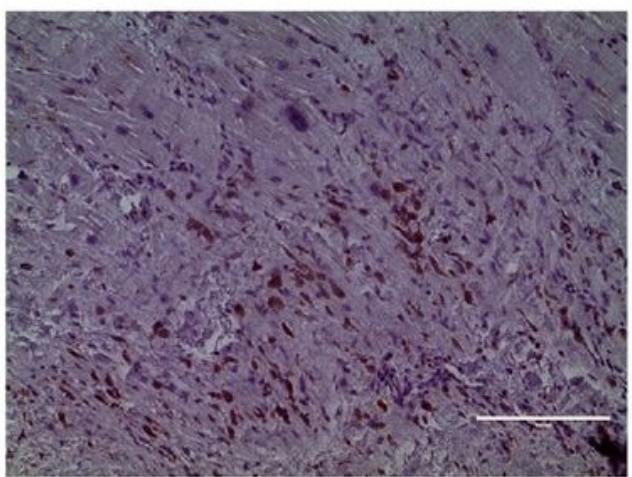

T2DM

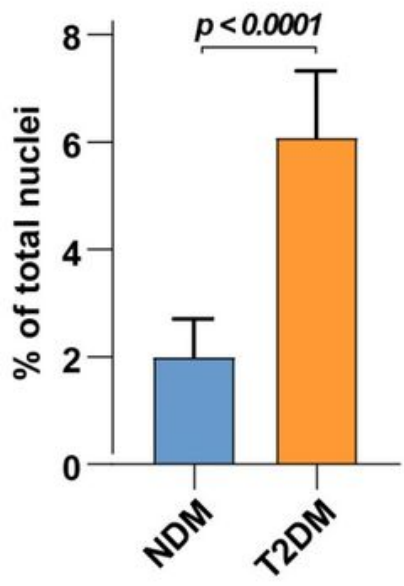

3-NT

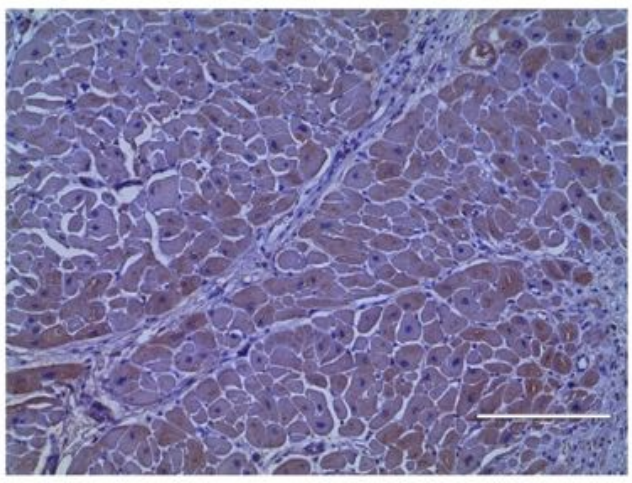

T2DM

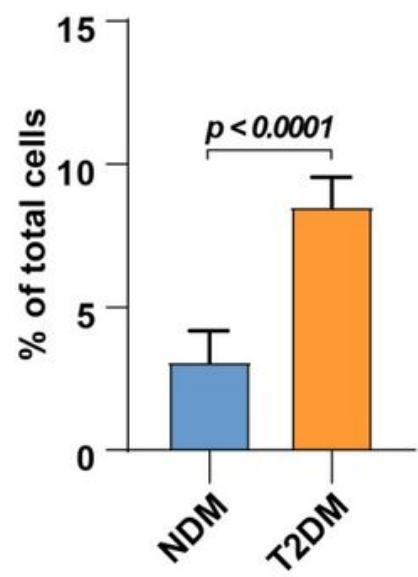

\section{4-HNE}

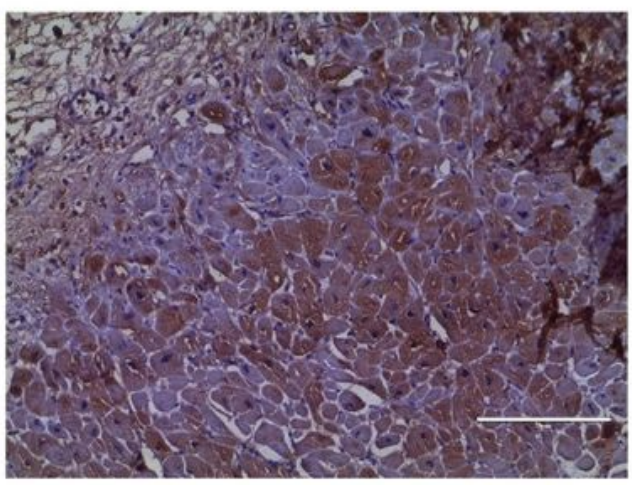

T2DM

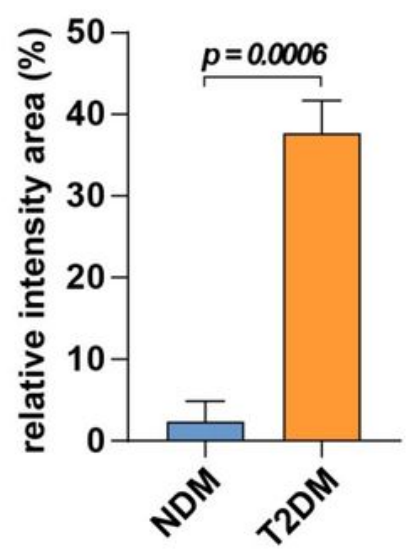

\section{Figure 1}

Cardiac damage in diabetes is associated with higher ROS production. (a-c) Light microscopy images show representative DAB-staining of LV samples obtained from T2DM and NDM patients. The respective bar graph show (a) the percentage of 8-OH-dG positive nuclei (in brown), (b) the percentage of 3-NT positive cells (in brown) and (c) the 4-HNE intensity levels (in brown) in T2DM cross section compared to NDM tissue sections (Representative of $n=3$ heart sections). Scale bar $=200 \mu \mathrm{m}$. All data are mean \pm S.D. 


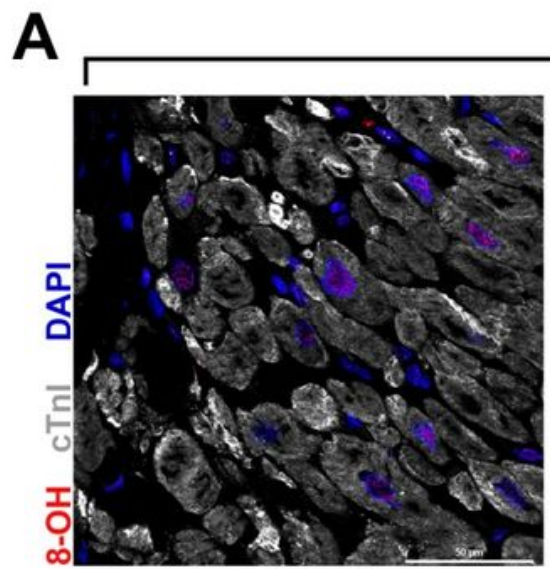

T2DM

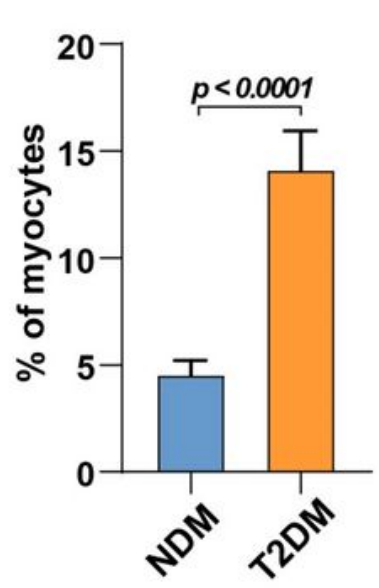

8-OH-dG

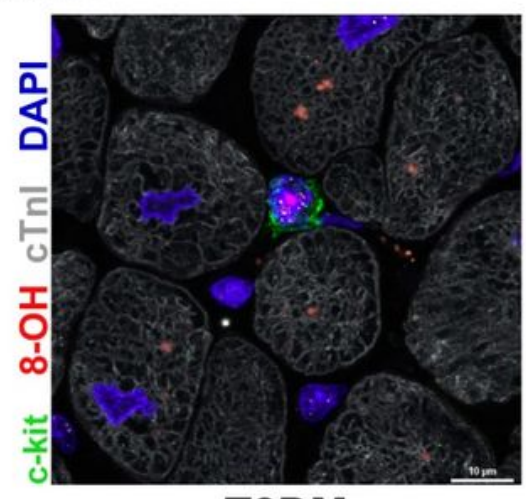

T2DM

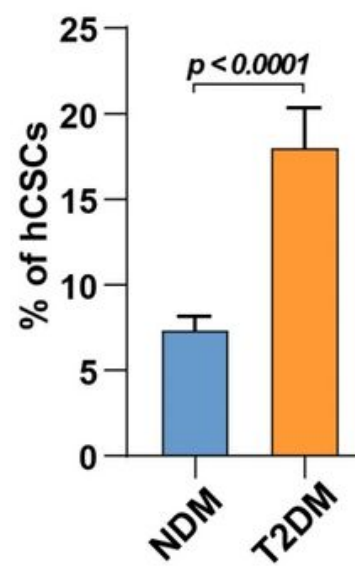

B

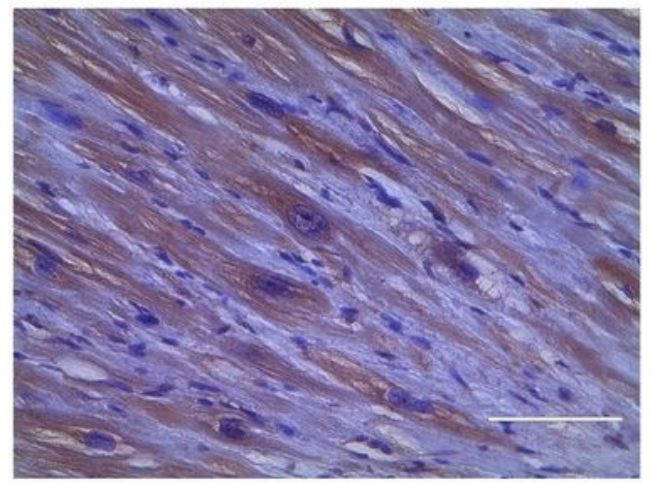

T2DM

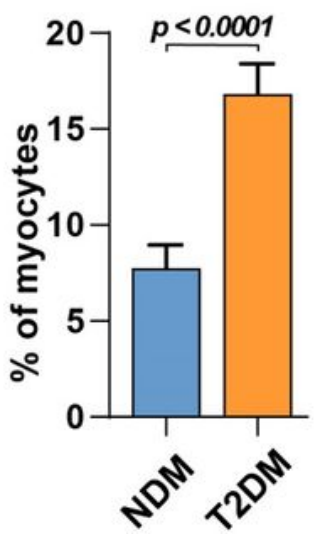

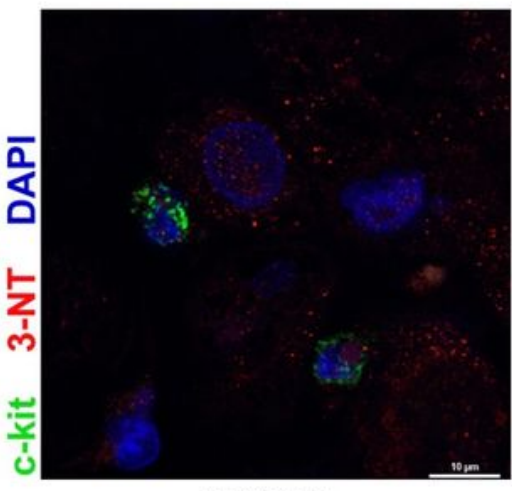

T2DM
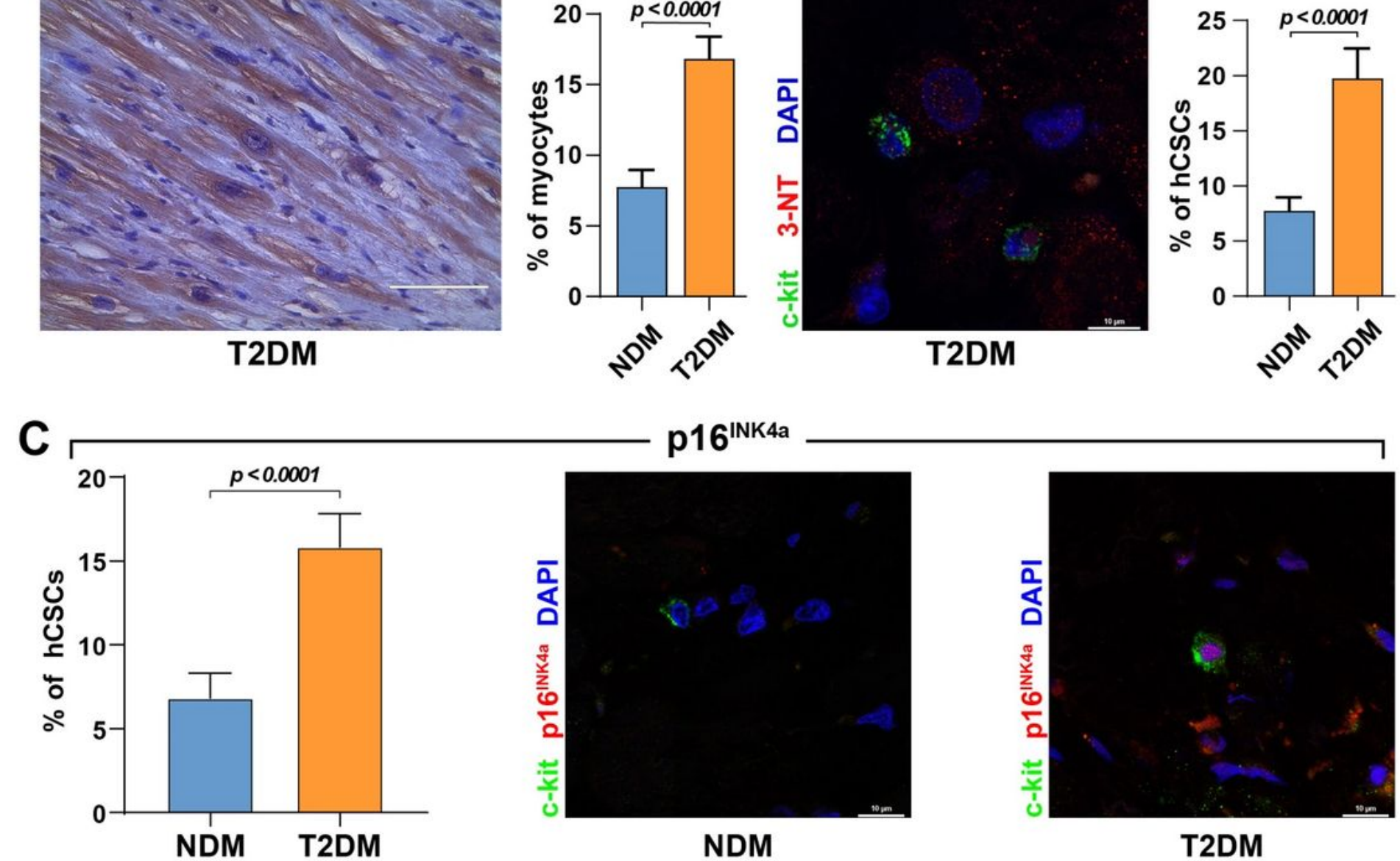

p16
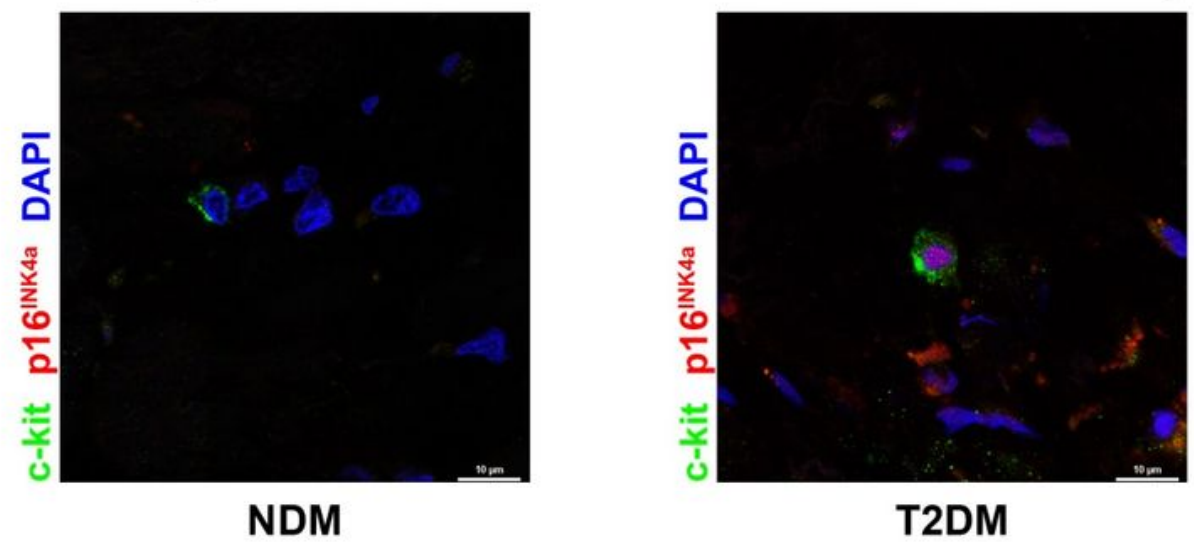

T2DM

Figure 2

Quantification of oxidative stress and senescent markers in myocytes and progenitor cells. (a)

Representative confocal images and bar graph showing 8-OH-dG positive cardiomyocytes and 8-OH-dG positive hCSCs in LV samples obtained from T2DM patients (8-OH-dG, red; c-kit, green; cTnl, white; DAPI, blue; representative of $n=3$ heart sections). Scale bar $=50 \mu \mathrm{m}$ and $10 \mu \mathrm{m}$ respectively (b) Representative light microscopy, bar graph and confocal images showing 3-NT positive cardiomyocytes (3-NT, brown) 
and 3-NT positive hCSCs in LV samples obtained from T2DM patients (3-NT, red; c-kit, green; DAPI, blue; representative of $n=3$ heart sections). Scale bar $=100 \mu \mathrm{m}$ and $10 \mu \mathrm{m}$ respectively. (c) Bar graph and representative confocal images showing p16INK4a expression in c-kitposCD45negCD31neg hCSCenriched cardiac cells in LV samples obtained from T2DM patients compared to NDM counterparts (p16INK4a, red; c-kit, green; DAPI, blue; representative of $n=3$ heart sections). Scale bar $=10 \mu m$. All data are mean \pm S.D.
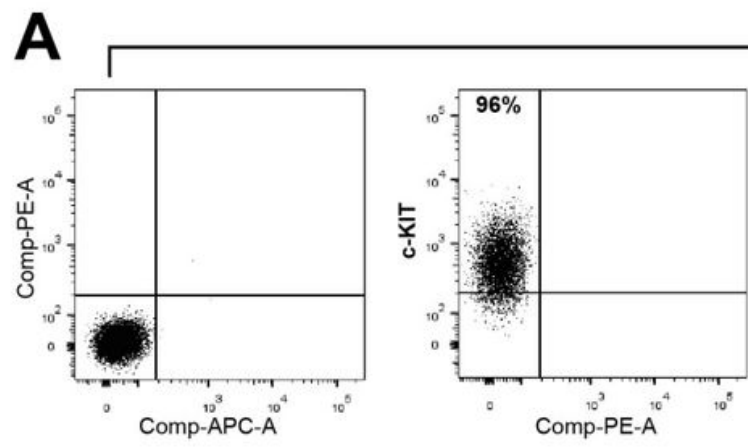

Diabetic hCSCs
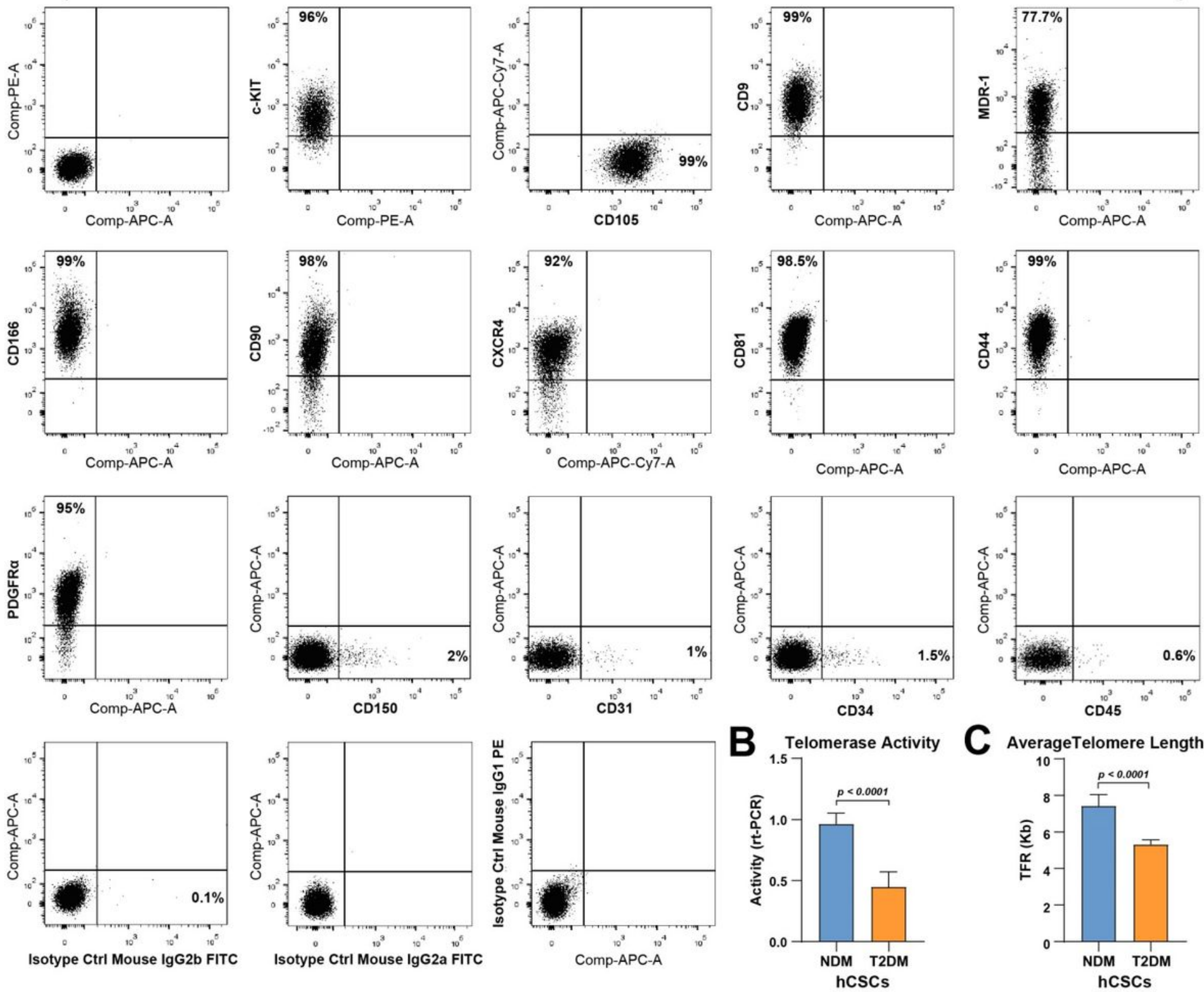

\section{Figure 3}

Phenotypic characterization of c-kitposCD45negCD31neg T2DM-hCSCs in vitro. (a) Flow cytometry dot plots showing the membrane phenotype of c-kitposCD45negCD31neg T2DM-hCSCs (Representative of $n=3$ number of biological replicates) $(b, c)$ Bar graphs showing a telomerase activity (b) and (c) average 
telomere length in c-kitposCD45negCD31neg T2DM-hCSCs compared to NDM-hCSCs. (Representative of $\mathrm{n}=3$ number of biological replicates). All data are mean \pm S.D.

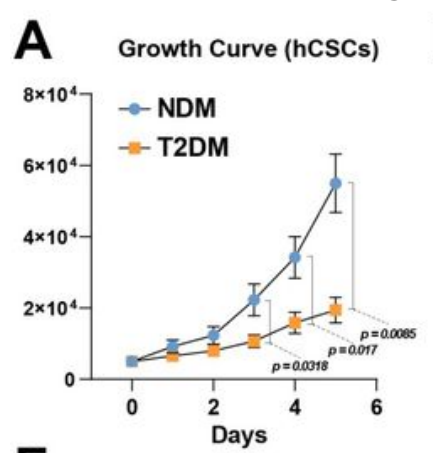

E
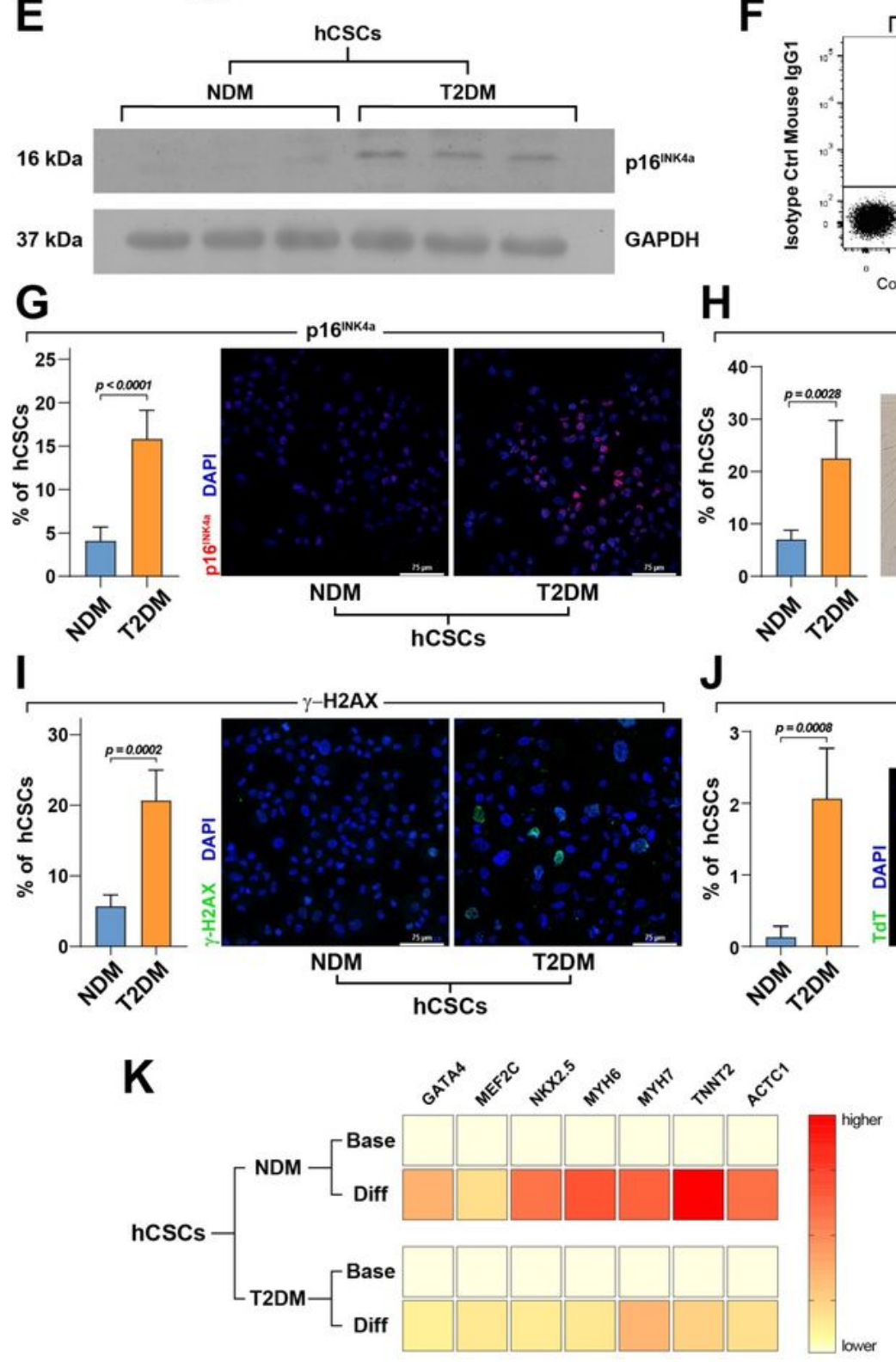

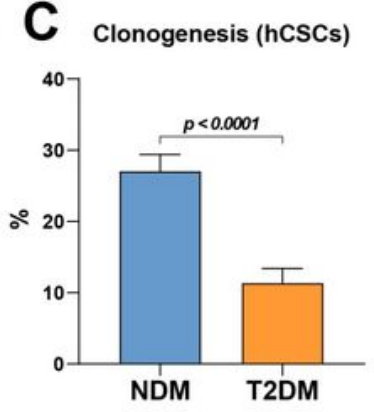

F
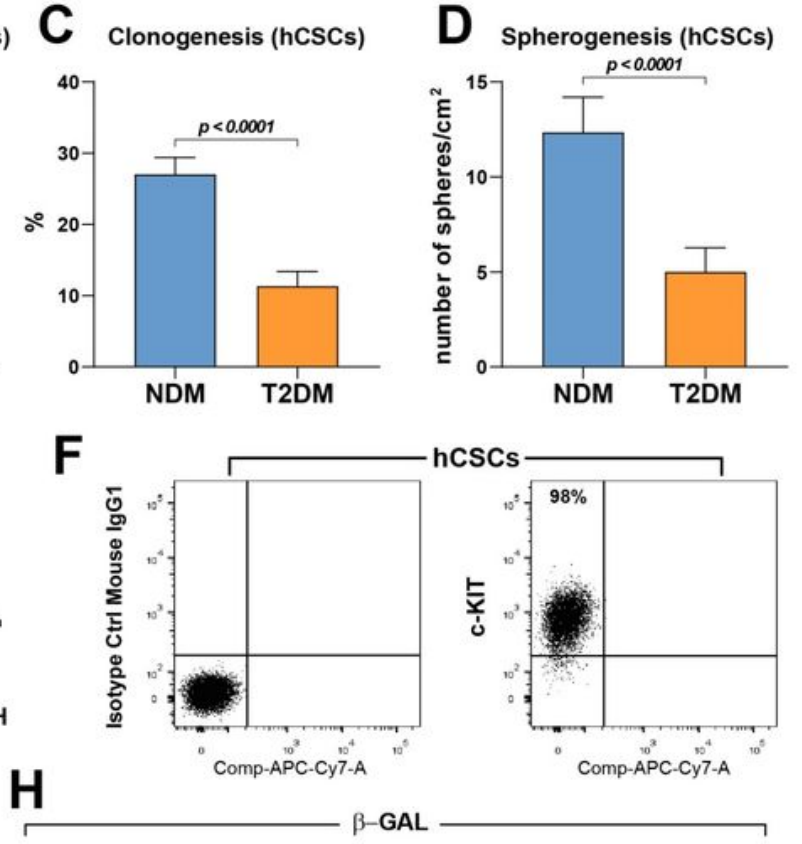

$\beta-G A L$

Apoptotic TdT*
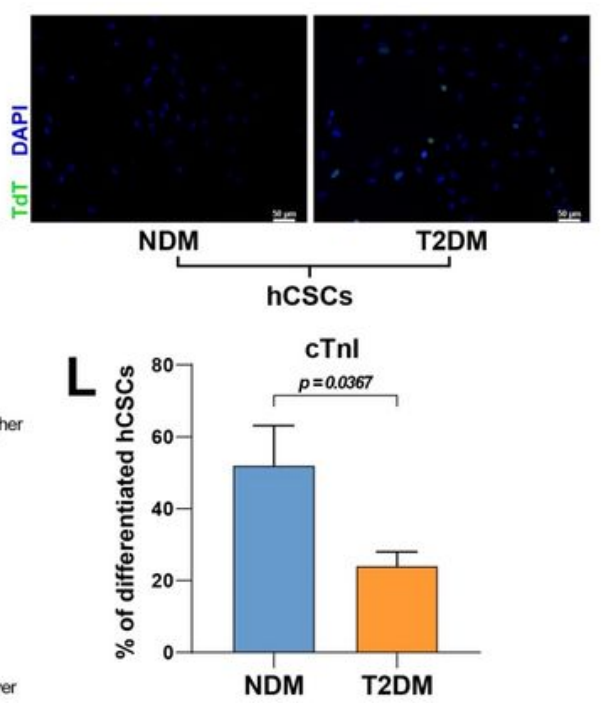

Figure 4

Impaired cell growth and myogenic differentiation potential of T2DM-hCSCs in vitro. (a) Cell growth curve shows a decreased proliferation in vitro of c-kitposCD45negCD31neg T2DM-hCSCs compared to NDMhCSCs (Representative of $n=3$ number of biological replicates). (b-d) Bar graphs showing respectively 
BrdU incorporation, clonogenesis and spherogenesis in c-kitposCD45negCD31neg T2DM-hCSCs compared to NDM-hCSCs (Representative of $n=3$ number of biological replicates). (e) Representative Western Blot showing p16INK4a levels in T2DM-hCSCs compared to NDM-hCSCs (Representative of $n=3$ biological replicates). (f) Flow cytometry dot plots showing a hCSCs population obtained from cardiomyocyte-depleted cardiac cell preparations. Left, representative gating strategy using isotype antibodies. Right, post-sorting flow cytometry dot plots showing c-kit expression in the cardiomyocytedepleted cardiac cell preparations negatively sorted for CD45 and CD31 (Representative of $n=3$ experiments). (g) Bar graph and representative confocal microscopy images from cytospin preparations showing p16INK4a expression (red) in c-kitposCD45negCD31neg T2DM-hCSCs compared to NDMhCSCs. Bar graph $=75 \mu \mathrm{m}$ (Representative of $\mathrm{n}=3$ biological replicates). (h) Bar graph and representative light microscopy images showing senescence-associated $\beta$-Gal positive cells (blue) in T2DM-hCSCs compared to NDM-hCSCs. Scale bar=200 $\mu \mathrm{m}$. (i,j) Bar graphs and representative confocal microscopy images from cytospin preparations of c-kitposCD45negCD31neg T2DM-hCSCs and NDM-hCSCs showing (i) the expression of $\mathrm{Y}-\mathrm{H} 2 \mathrm{AX}$, (green) and (j) TdT (green) in T2DM-hCSCs compared to NDM-hCSCs. Bar graph $=75 \mu \mathrm{m}$ and $50 \mu \mathrm{m}$. (Representative of $\mathrm{n}=3$ biological replicates). (k) Heat maps showing qRT- PCR analysis of the main cardiac transcription factors and myocyte contractile genes in ckitposCD45negCD31neg T2DM-hCSCs compared with NDM-hCSCs 14 days after myogenic differentiation induction. Color scale indicates change in $\mathrm{Ct}$ (threshold cycle) relative to the normalized GAPDH control (Representative of $n=3$ biological replicates). (I) Bar graph showing cTnl expression levels of differentiated T2DM-hCSCs compared to NDM-hCSCs (Representative of $n=3$ biological replicates). All data are mean $\pm S . D$ 

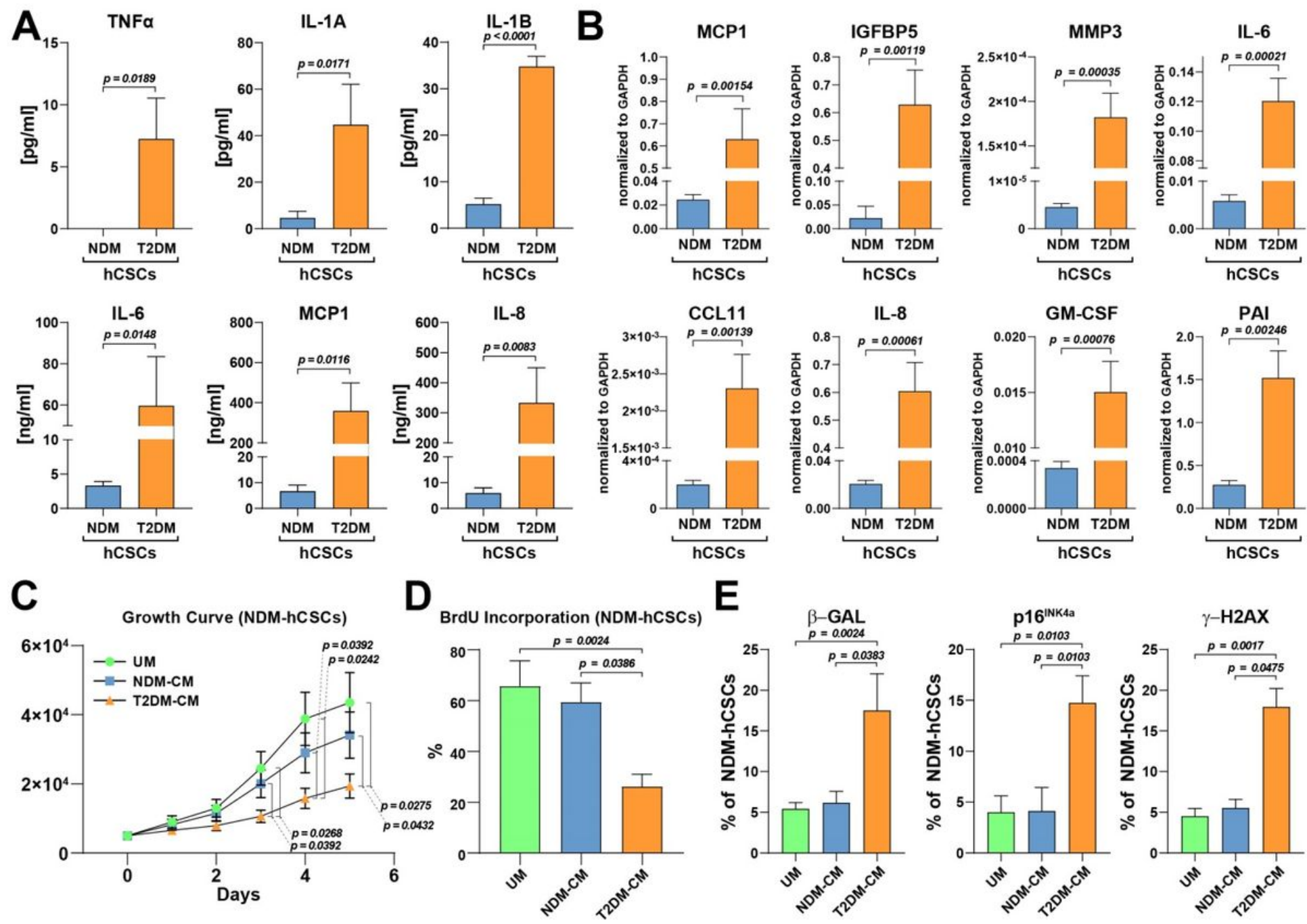

Figure 5

The senescence-associated secretory phenotype (SASP) of T2DM-hCSCs. (a) Bar graphs showing the SASP factor protein levels in culture media from T2DM-hCSCs and NDM-hCSCs after 24 hours in serumfree conditions (Representative of $n=3$ biological replicates). (b) Bar graphs showing transcript SASP factors expression in T2DM-hCSCs vs. NDM-hCSCs (Representative of $n=3$ biological replicates). (c) Cell growth curve showing the in vitro proliferative change of NDM-hCSCs placed in conditioned medium (CM) derived from T2DM-hCSCs compared to the NDM-hCSCs placed in unconditioned medium (UM) and NDM-hCSCs CM (Representative of $n=3$ number of biological replicates). (d) Bar graph showing BrdU incorporation of NDM-hCSCs placed in UM, NDM-CM and T2DM-CM (Representative of $n=3$ biological replicates). (e) Bar graphs showing senescent p16INK4a positive hCSCs, SA- $\beta$-gal-positive hCSCs and $\gamma^{-}$ H2AX-positive hCSCs in NDM-hCSCs treated with T2DM-CM compared to UM and NDM-CM (Representative of $n=3$ number of biological replicates). All data are mean \pm S.D 


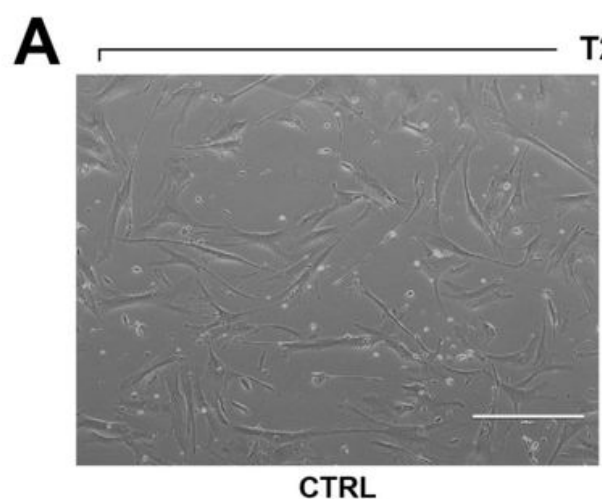

\section{CTRL}
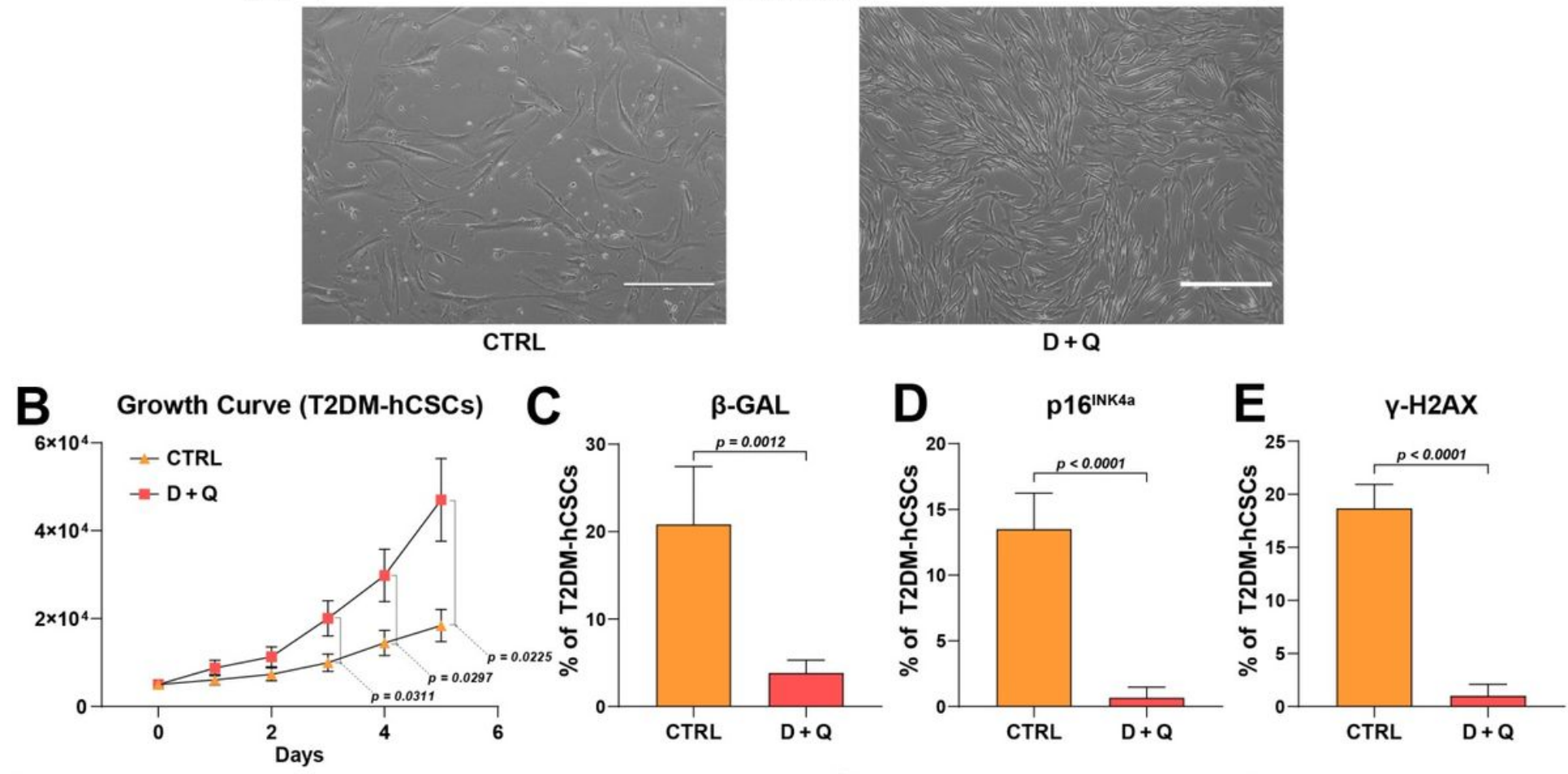
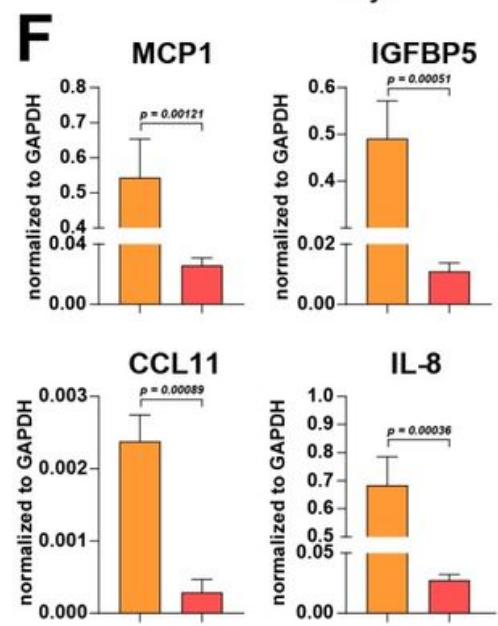

$\square$ CTRL (T2DM-hCSCs)
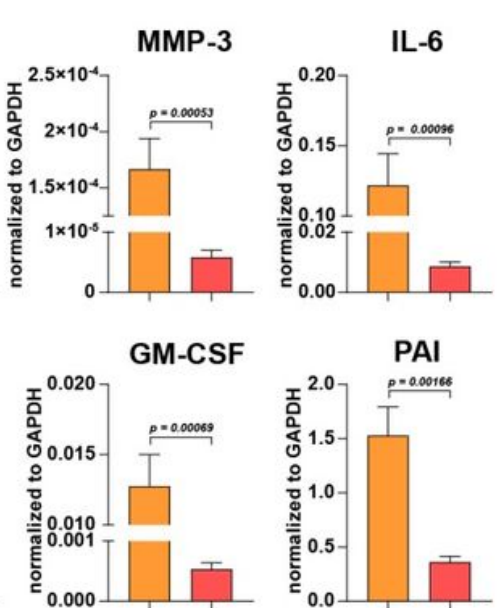

D + Q (T2DM-hCSCs)

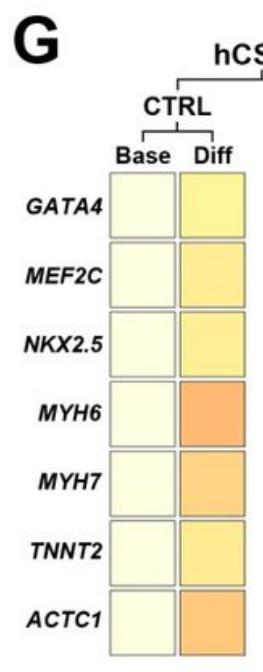

hcscs

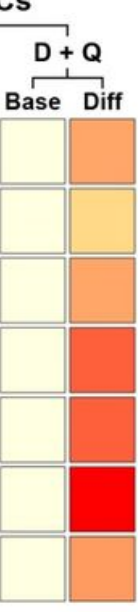

$\mathbf{H}$

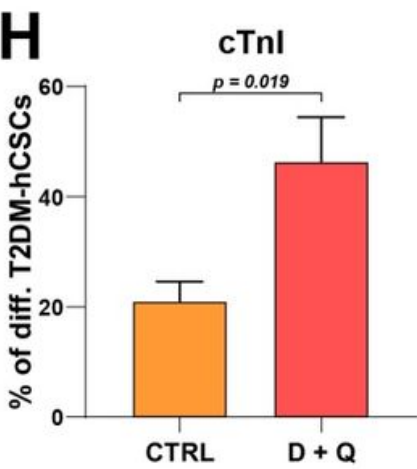

\section{Figure 6}

Senolytics ameliorates regenerative deficit of diabetic hCSCs. (a) Light microscopy images showing that the typical enlarged and flattened morphology of senescent cells present in untreated control (CTRL)

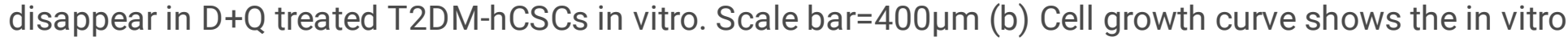
proliferation of c-kitposCD45negCD31neg T2DM-hCSCs after D+Q treatment compared to CTRL per se (Representative of $n=3$ number of biological replicates). (c-e) Bar graphs showing the number of (c) SA- $\beta$ gal, (d) p16INK4a senescent cells and (e) $\mathrm{Y}$-H2AX positive cells in T2DM-hCSCs after D+Q treatment compared to CTRL (Representative of $n=3$ number of biological replicates). (f) Bar graphs showing transcript SASP factors expression in T2DM-hCSCs after D+Q treatment vs. CTRL (Representative of $n=3$ biological replicates). (g) Heat maps showing qRT-PCR analysis of the main cardiac transcription factors and myocyte contractile genes (GATA-4, NKX2.5, MEF2C, TNNT2, ACTC1, MYH6 and MYH7) in 
differentiating T2DM-hCSCs after D+Q treatment vs. CTRL. Color scale indicates change in Ct (threshold cycle) relative to the normalized GAPDH control (Representative of $n=3$ biological replicates). (h) Bar graph show cTnl expression levels in differentiated T2DM-hCSCs after D+Q treatment vs. CTRL (Representative of $n=3$ biological replicates). All data are mean \pm S.D 